\title{
Multiantenna GLR Detection of Rank-One Signals with Known Power Spectral Shape under Spatially Uncorrelated Noise
}

\author{
Josep Sala-Alvarez†, Gonzalo Vázquez-Vilarł, Roberto López-Valcarceł†, Saeid Sedighił‡, Abbas Taherpourł‡
}

\begin{abstract}
We establish the generalized likelihood ratio (GLR) test for a Gaussian signal of known power spectral shape and unknown rank-one spatial signature in additive white Gaussian noise with an unknown diagonal spatial correlation matrix. This is motivated by spectrum sensing problems in dynamic spectrum access (DSA), in which the temporal correlation of the primary signal can be assumed known up to a scaling, and where the noise is due to an uncalibrated receive array. For spatially independent identically distributed (i.i.d.) noise, the corresponding GLR test reduces to a scalar optimization problem, whereas the GLR detector in the general non-i.i.d. case yields a more involved expression, which can be computed via alternating optimization methods. Low signal-to-noise ratio (SNR) approximations to the detectors are given, together with an asymptotic analysis showing the influence on detection performance of the signal power spectrum and SNR distribution across antennas. Under spatial rank- $P$ conditions, we show that the rank-one GLR detectors are consistent with a statistical criterion that maximizes the output energy of a beamformer operating on filtered data. Simulation results support our theoretical findings in that exploiting prior knowledge on the signal power spectrum can result in significant performance improvement.
\end{abstract}

Index Terms-GLRT, detection, spectrum sensing, cognitive radio, multiple antenna, uncalibrated array.

\section{INTRODUCTION}

Array processing for signal detection and parameter estimation has drawn great research interest, and a rich corpus of techniques has been developed so far for scenarios in additive Gaussian noise, e.g. [1]-[6]. Apart from some approaches based on correlation matching for single-antenna receivers, e.g. [7]-[10], most works do not assume prior knowledge on the shape of the power spectral density (PSD) of the signal of interest. In fact, the exploitation of an additional temporal dimension on a sound statistical basis leads to substantial difficulties if exact expressions are sought. For instance, [11] relied on a low signal-to-noise ratio (SNR) approximation to the Neyman-Pearson detector to obtain practical detectors for spatially rank-one signals under spatially white noise of known

Copyright (c) 2016 IEEE. Personal use of this material is permitted However, permission to use this material for any other purposes must be obtained from the IEEE by sending a request to pubs-permissions@ieee.org.

The authors are with the: †Dept. of Signal Theory and Communications, Universitat Politècnica de Catalunya-Barcelona TECH (UPC), 08034 Barcelona, SPAIN (josep.sala@upc.edu); †Dept. of Signal Theory and Communications, Universidad Carlos III de Madrid (UC3M), 28911 Leganés, SPAIN (gonzalo.vazquez@uc3m.es); $\quad$ † Dept. of Signal Theory and Communications, University of Vigo, 36310 Vigo,

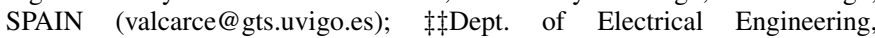
Imam Khomeini International University (IKIU), Qazvin, IRAN ( \{s.sedighi,taherpour\}@eng.ikiu.ac.ir, \{saeidsedighi,taherpour\}@ gmail.com).

Work supported by the "Ministerio de Economía y Competitividad" of the Spanish Government and the European Regional Development Fund (ERDF) (TEC2013-47020-C2-1-R and TEC2013-47020-C2-2-R COMPASS, TEC2013-41718-R, FPDI-2013-18602 and TEC2015-69648-REDC Red COMONSENS), the Catalan Government (2014 SGR 60 AGAUR) and the Galician Government and ERDF (GRC2013/009, R2014/037 REdTEIC and AtlantTIC). variance, whereas [12] derived the exact generalized likelihood ratio test (GLRT) under spatially unstructured noise with an unknown spatial correlation matrix. In this work, we obtain the exact GLRT for the detection of signals with a known PSD shape and spatial rank one in spatially structured noise. Specifically, we assume an unknown diagonal form for the noise spatial correlation matrix, either with equal diagonal elements (calibrated receiver) or possibly different (uncalibrated receiver). The mathematical approach proves substantially different from that in [11] when aiming at unknown instead of known per-antenna noise variances, together with exact all-SNR rather than approximate low-SNR detectors. With respect to [12], considering an unknown diagonal form for the noise spatial correlations leads to fundamental differences in terms of approach, structure of the detector and improved performance.

The model under study emerges in the context of dynamic spectrum access (DSA) in licensed bands [13], [14]. DSA seeks a more efficient usage of the available spectrum by allowing access of unlicensed (secondary) users while limiting interference to licensed (primary) users. This requires secondary users to have spectrum sensing capabilities [1] as well as access policies [15] for exploiting spectrum holes. Important effects such as large/small-scale fading, the hidden node problem, noise uncertainty, low SNR operation, etc., appear in the design and analysis of suitable detection schemes, and may be tackled, albeit with different effectivity, via cooperative detection (with limited data exchanges between sensors) and multiantenna detection (with centralized access to all data samples).

Focusing on the latter, multiantenna detection research has exploited different aspects: (i) low-rank spatial correlation matrix of the primary signal; (ii) noise spatial correlation structure; (iii) other signal features, which may be regarded as random, unknown deterministic, or known. The abundance of scenarios subject to parameter uncertainties has resulted in widespread application of the GLRT to avoid detector degradation. Detection of spatially rank-one unknown deterministic signals is addressed in [16] and [17] under spatially i.i.d. noise of unknown variance, and in [2] for uncalibrated receivers. For random signals the rank-one case is examined in [4], [18] for signals with a white spectrum, and in [11], [12] for known temporal correlation. Wideband rank-one signal detection is treated in [19] under spatially uncorrelated noise.

Our scenario of interest is the co-existence of secondary users equipped with multiantenna transceivers and a primary network using frequency-division multiple access, i.e., splitting its allocated bandwidth into a number of low-bandwidth frequency channels. The secondary users perform frequency scanning by tuning into each possible primary channel to detect idle frequency slots for transmission. Over each specific 
Copyright (c) 2016 IEEE. Personal use of this material is permitted. However, permission to use this material for any other purposes must be obtained from the IEEE by sending a request to pubs-permissions@ieee.org.

frequency slot, the delay spread is much lower than the symbol period, so that the channel from the primary transmitter to each secondary user antenna can be regarded as flat-fading. The primary transmitter is equipped with a single antenna, and a spatial rank-one signal model naturally emerges at the secondary multiantenna receiver. As we shall see, detectors derived under this model correspond to beamformers steered to collect the maximum energy from the spatial components of the received signal, and hence they are robust to signals with spatial rank larger than one. In fact, the information on the power spectral shape is employed for spectral weighting of the received data at the input to the beamformer when computing the corresponding sample spatial correlation matrix. It is worth noting that several GLR detectors have also been derived in [20] for signals with spatial rank $P \geq 1$ in spatially white noise of known and unknown variance. These detectors, though, do not exploit prior knowledge on the power spectral shape.

We intend to derive detectors applicable to a broad class of signals, which: (i) capture essential features of the signal model, rather than rely on detailed specificities of the modulation used; (ii) nonetheless attain good performance over this class; and (iii) are computationally feasible. Additionally, we seek to exploit a fundamental feature of communication signals, the power spectral density (PSD). In the described scenario, the normalized PSD or emission mask of primary signals is publicly known and it can be assumed available to the spectral monitor. The primary signal PSD is in fact related to the temporal correlation matrix of the signal, while it does not depend on the complementary non-zero correlations exhibited by improper (non-circularly-symmetric) signals. Hence, and without precluding other approaches, both signal and noise are assumed zero-mean complex circularly-symmetric Gaussian. Altogether, the detectors derived under this model achieve the conditions (i)-(iii) even if the signal is nonGaussian and/or improper, as will be shown via simulations.

At reception, tolerances in the components of the different radio-frequency $(\mathrm{RF})$ chains originate different per-antenna noise power levels. In fact, several reported detectors are sensitive to these calibration errors [6]. Here, we consider scenarios in which the noise is spatially white (calibrated receiver), or possibly has distinct noise levels at each antenna (uncalibrated receiver). When the noise is independent identically distributed (i.i.d.), the GLRT boils down to a scalar optimization problem, whereas for spatially non-i.i.d. noise, a more involved process is required. In the low-SNR regime the GLRT is expressed in closed-form under both models, and it is shown to yield the exact all-SNR GLRT under certain conditions.

Although our main motivation is rooted in DSA scenarios, the detectors discussed in this paper may find application in other areas as well. For example, in passive radar systems, target detection exploits readily available, non-cooperative illuminators of opportunity such as broadcast radio or television transmissions [21]-[23]. In such systems, the transmitted signal is out of control and generally unknown to a passive receiver, and a noisy reference may or may not be available [24], [25]. However, the normalized PSD of the transmitted signal may be known, and it would make sense to exploit this feature to improve detection. At the same time, noise calibration issues become important as well in passive radar systems.

Organization: Sec. II introduces the signal model and the problem formulation. In Sec. III we derive the GLR detectors under both noise models and we address their asymptotic performance analysis in Sec. IV. The GLR detectors are particularized under certain signal models in Sec. V. Sec. VI presents comparative simulation results and Sec. VII concludes the paper.

Notation: Bold lower-/upper-case denote vectors/matrices. $\boldsymbol{A}^{*}, \boldsymbol{A}^{T}, \boldsymbol{A}^{H}$ and $\boldsymbol{A}^{-1}$ stand for the conjugate, transpose, conjugate transpose and inverse of matrix $\boldsymbol{A}$. $\mathbf{I}_{n}$ and $\mathbf{0}_{n \times m}$ are the $n \times n$ identity and $n \times m$ zero matrices. The reduced Singular Value Decomposition (SVD) of rank- $r \boldsymbol{A} \in \mathbb{C}^{n \times m}$ is $\boldsymbol{A}=\boldsymbol{U} \boldsymbol{S} \boldsymbol{V}^{H}$, with $\boldsymbol{U} \in \mathbb{C}^{n \times r}, \boldsymbol{V} \in \mathbb{C}^{m \times r}$ semi-unitary and $\boldsymbol{S} \in \mathbb{C}^{r \times r}$ diagonal positive-definite. $\otimes$ is Kronecker's product and $\operatorname{tr}(\cdot), \operatorname{det}(\cdot)$ the trace and determinant. For vector $\mathbf{x}, \operatorname{diag}(\mathbf{x})$ is its associated diagonal matrix; for matrix $\mathbf{X}, \operatorname{diag}(\mathbf{X})$ is a diagonal matrix with diagonal that of $\mathbf{X}$. $\lambda_{\max }[\boldsymbol{A}]$ and $\boldsymbol{e}_{\max }[\boldsymbol{A}]$ (resp. $\lambda_{\min }[\boldsymbol{A}]$ and $\boldsymbol{e}_{\min }[\boldsymbol{A}]$ ) denote the maximum (resp. minimum) eigenvalue of $\boldsymbol{A}$ and its associated eigenvector. $\mathbb{E}[\cdot]$ is the statistical expectation, log is base- $e$ logarithm, $o(\cdot)$ Landau's small-o, and $[x]^{+} \doteq \max \{0, x\}$.

\section{Problem Formulation}

The sensor has $M$ antennas with their respective $\mathrm{RF}$ chains. The same primary channel is selected at all antennas, downconverted to baseband and its I\&Q channels are sampled at rate $f_{s}=1 / T_{s}$. The $M$ complex samples at time $k T_{s}$ are arranged into the vector $\mathbf{y}_{k}=s_{k} \mathbf{h}+\mathbf{n}_{k}$, with $\mathbf{h} \doteq\left[h_{1}, \cdots, h_{M}\right]^{T}$ the vector of complex channel gain coefficients, $s_{k} \doteq s\left(k T_{s}\right)$ the transmitted baseband signal sample, and $\mathbf{n}_{k} \doteq\left[n_{1}\left(k T_{s}\right), \cdots, n_{M}\left(k T_{s}\right)\right]^{T}$ the received complex noise vector. Using Kronecker's product, we stack $N$ consecutive array snapshots $\mathbf{y}_{k}$ to express the received signal,

$$
\mathbf{y}=\mathbf{s} \otimes \mathbf{h}+\mathbf{n},
$$

with $\mathbf{s} \doteq\left[s_{1}, \cdots, s_{N}\right]^{T}$ the temporal transmitted signal vector and $\mathbf{y} \doteq\left[\mathbf{y}_{1}^{T}, \cdots, \mathbf{y}_{N}^{T}\right]^{T}$ and $\mathbf{n} \doteq\left[\mathbf{n}_{1}^{T}, \cdots, \mathbf{n}_{N}^{T}\right]^{T}$ the received signal and noise stacked vectors, respectively. This model assumes that over the RF channel bandwidth the channel from the primary transmitter to the spectrum monitor is frequencyflat and static during a sensing interval of duration $N T_{s}$. Following [26], it is also assumed that no secondary users transmit during the sensing period.

Both $\mathbf{s}$ and $\mathbf{n}$ are assumed zero-mean complex circularlysymmetric and Gaussian. The Gaussianity assumption is a common one [27]-[29] and usually leads to estimators/detectors that operate on sufficient statistics which happen to be sample moments of the data y [30], [31] (more precisely, proper sample moments under the circular symmetry assumption). For specific signal types such as Orthogonal Frequency Division Multiplexing (OFDM), the Gaussianity assumption constitutes a good approximation if the number of subcarriers is large. Moreover, it has been shown that, at low SNR, a Gaussian distribution with second-order moments matching those of the true distribution is a valid approach for exploiting correlation features in certain estimation problems [31]. 
Copyright (c) 2016 IEEE. Personal use of this material is permitted. However, permission to use this material for any other purposes must be obtained from the IEEE by sending a request to pubs-permissions@ieee.org.

We let $\mathbf{C} \doteq \mathbb{E}\left[\mathbf{s s}^{H}\right]$ denote the $N \times N$ temporal correlation matrix of the primary signal with normalized trace $\frac{1}{N} \operatorname{tr} \mathbf{C}=$ 1. This matrix is directly related to the primary signal PSD $P_{s}(f)$, which is assumed known to the spectral monitor as motivated in Section I. In fact, if we let $\mathbf{C}=\mathbf{U} \boldsymbol{\Lambda} \mathbf{U}^{H}$ be an eigendecomposition of $\mathbf{C}$ with $\boldsymbol{\Lambda}=\operatorname{diag}\left(\left[\lambda_{0} \lambda_{1} \cdots \lambda_{N-1}\right]^{T}\right)$ and $\mathbf{U} N \times N$ unitary, then it is well known [32], [33] that as $N \rightarrow \infty$ the matrix of eigenvectors $\boldsymbol{U}$ approaches the $N \times N$ orthonormal inverse discrete Fourier transform (IDFT) matrix, whereas the eigenvalues satisfy

$$
\lambda_{k} \rightarrow P_{s}(k / N), \quad 0 \leq k \leq N-1 .
$$

The normalization $\frac{1}{N} \operatorname{tr}(\mathbf{C})=1$ implies that $\int_{0}^{1} P_{s}(f) \mathrm{d} f=1$.

The noise $\mathbf{n}$ is modeled as temporally white Gaussian, statistically independent of $\mathbf{s}$. The $M \times M$ spatial correlation matrix of the noise process is denoted as $\boldsymbol{\Sigma}^{2} \doteq \mathbb{E}\left[\mathbf{n}_{k} \mathbf{n}_{k}^{H}\right]$. Then, the $M N \times M N$ data covariance matrix becomes

$$
\mathbf{R}_{y} \doteq \mathbb{E}\left[\mathbf{y} \mathbf{y}^{H}\right]=\mathbf{I}_{N} \otimes \mathbf{\Sigma}^{2}+\mathbf{C} \otimes \mathbf{h h}^{H} .
$$

Although estimating noise power at each antenna in an initial calibration stage is feasible in the absence of interference, noise uncertainty is unavoidable due to calibration errors, temperature drifts or component aging. This effect is manifest in terms of an SNR wall [34], below which detection degrades, regardless of the duration of the sensing interval. In this respect, it is of interest to develop detectors that do not require precise knowledge of the noise spatial correlation matrix $\boldsymbol{\Sigma}^{2}$.

We will conduct a comparative analysis under the following models for $\Sigma^{2}$ :

- Model 1: Calibrated system with equal noise powers at each of the antennas. This system corresponds to spatially i.i.d. (or spatially white) noise, i.e.,

$$
\boldsymbol{\Sigma}^{2}=\sigma^{2} \mathbf{I}_{M}
$$

This model was considered in [11] under the assumption of a known noise variance $\sigma^{2}$, and in [4] for $\mathbf{C}=\mathbf{I}_{N}$ (temporally white signal) and $\sigma^{2}$ unknown.

- Model 2: System with uncalibrated RF front-ends or when receiver antennas are not co-located. This system corresponds to spatially uncorrelated noise and it is modeled by $\boldsymbol{\Sigma}^{2}$ being a diagonal matrix, i.e.,

$$
\boldsymbol{\Sigma}^{2}=\operatorname{diag}\left(\left[\sigma_{1}^{2}, \ldots, \sigma_{M}^{2}\right]^{T}\right) .
$$

This model was considered in [3], [6] for $\mathbf{C}=\mathbf{I}_{N}$.

- Model 3: Spatially correlated noise or broadband interference $\left(\Sigma^{2}\right.$ an arbitrary full-rank positive definite matrix). This model has been treated in [12] and will be used here only in comparison with the previous models.

From the Gaussian assumption, the parametrized probability density function (p.d.f.) of the data in terms of the covariance matrix $\mathbf{R}_{y}$ is expressed as follows, with the dependence on the unknown parameters $\mathbf{h}$ and $\boldsymbol{\Sigma}^{2}$ implicit in $\mathbf{R}_{y}$ as in (3),

$$
f\left(\mathbf{y} \mid \mathbf{R}_{y}\right)=\frac{\exp \left(-\mathbf{y}^{H} \mathbf{R}_{y}^{-1} \mathbf{y}\right)}{\pi^{M N} \operatorname{det} \mathbf{R}_{y}},
$$

Our problem is to determine the presence or absence of the signal from an observation $\mathbf{y}$. The corresponding hypothesis test can be written in terms of the covariance matrix $\mathbf{R}_{y}$ as

$$
\begin{aligned}
& \mathcal{H}_{0}: \mathbf{R}_{y}=\mathbf{I}_{N} \otimes \boldsymbol{\Sigma}^{2} \doteq \mathbf{R}_{0} \\
& \mathcal{H}_{1}: \mathbf{R}_{y}=\mathbf{I}_{N} \otimes \boldsymbol{\Sigma}^{2}+\mathbf{C} \otimes \mathbf{h h}^{H} \doteq \mathbf{R}_{1} .
\end{aligned}
$$

Since $\mathbf{h}$ and $\boldsymbol{\Sigma}^{2}$ are unknown, a sensible approach is the GLRT. In the GLRT, the unknown parameters in the likelihood ratio are substituted by their Maximum Likelihood (ML) estimates under each hypothesis, yielding

$$
T \doteq \frac{\max _{\mathbf{R}_{1}} f\left(\mathbf{y} \mid \mathbf{R}_{1}\right)}{\max _{\mathbf{R}_{0}} f\left(\mathbf{y} \mid \mathbf{R}_{0}\right)} \underset{\mathcal{H}_{0}}{\stackrel{\mathcal{H}_{1}}{\gtrless}} \gamma,
$$

for a suitable threshold $\gamma>1$. This test will be established under Models 1, 2 and 3 for $\boldsymbol{\Sigma}^{2}$. Note that $\boldsymbol{\Sigma}^{2}$ under model $m$ subsumes $\boldsymbol{\Sigma}^{2}$ under models $m^{\prime}<m$. Hence, its corresponding detector will be capable to operate under the previous noise models (although with possibly different performance).

\section{GLR DETECTORS}

To derive the ML estimates under both hypotheses, we will first find an alternative expression for the log-likelihood,

$$
\log f\left(\mathbf{y} \mid \mathbf{R}_{y}\right)=-\mathbf{y}^{H} \mathbf{R}_{y}^{-1} \mathbf{y}-M N \log \pi-\log \operatorname{det} \mathbf{R}_{y} .
$$

We will operate only with $\mathbf{R}_{y}=\mathbf{R}_{1}$ under $\mathcal{H}_{1}$, as, setting $\mathbf{h}=\mathbf{0}_{M}$, yields the corresponding expressions under $\mathcal{H}_{0}$.

Using a generalization of Sylvester's determinant identity to Kronecker products [35], in view of (3), we can rewrite the determinant term in (10) as

$$
\log \operatorname{det} \mathbf{R}_{y}=N \log \operatorname{det}\left(\boldsymbol{\Sigma}^{2}\right)+\log \operatorname{det}\left(\mathbf{I}_{N}+\rho \mathbf{C}\right),
$$

where the parameter $\rho \doteq \mathbf{h}^{H} \boldsymbol{\Sigma}^{-2} \mathbf{h}$ corresponds to the SNR at the output of the Capon beamformer $\mathbf{w} \doteq \boldsymbol{\Sigma}^{-2} \mathbf{h}$ when signal $\mathbf{s}$ is unit-power. The inverse of the received signal covariance matrix can also be written as [12],

$$
\begin{aligned}
& \mathbf{R}_{y}^{-1}=\mathbf{I}_{N} \otimes \boldsymbol{\Sigma}^{-2}-\mathbf{C}(\rho) \otimes\left(\boldsymbol{\Sigma}^{-2} \mathbf{h} \mathbf{h}^{H} \boldsymbol{\Sigma}^{-2}\right), \\
& \mathbf{C}(\rho) \doteq \mathbf{C}\left(\mathbf{I}_{N}+\rho \mathbf{C}\right)^{-1} .
\end{aligned}
$$

Then, using $\operatorname{tr}\left(\mathbf{A}^{T} \mathbf{B}^{H} \mathbf{C} \mathbf{D}\right)=\operatorname{vec}(\mathbf{B})^{H}(\mathbf{A} \otimes \mathbf{C}) \operatorname{vec}(\mathbf{D})$, straightforward algebra yields,

$$
\begin{aligned}
& \mathbf{y}^{H} \mathbf{R}_{1}^{-1} \mathbf{y} \\
& \quad=\operatorname{tr}\left(\boldsymbol{\Sigma}^{-2} \mathbf{Y} \mathbf{Y}^{H}\right)-\operatorname{tr}\left(\boldsymbol{\Sigma}^{-2} \mathbf{h} \mathbf{h}^{H} \boldsymbol{\Sigma}^{-2} \mathbf{Y} \mathbf{C}^{*}(\rho) \mathbf{Y}^{H}\right),
\end{aligned}
$$

with $\mathbf{Y} \doteq\left[\mathbf{y}_{1} \cdots \mathbf{y}_{N}\right]$ the $M \times N$ received data matrix. Substituting (11) and (14), the log-likelihood (10) becomes,

$$
\begin{aligned}
& \log f\left(\mathbf{Y} \mid \mathbf{h}, \boldsymbol{\Sigma}^{2}\right) \\
& =-M N \log \pi-N \log \operatorname{det} \boldsymbol{\Sigma}^{2}-\log \operatorname{det}\left(\mathbf{I}_{N}+\rho \mathbf{C}\right) \\
& -\operatorname{tr}\left(\boldsymbol{\Sigma}^{-2} \mathbf{Y} \mathbf{Y}^{H}\right)+\operatorname{tr}\left(\boldsymbol{\Sigma}^{-2} \mathbf{h} \mathbf{h}^{H} \boldsymbol{\Sigma}^{-2} \mathbf{Y} \mathbf{C}^{*}(\rho) \mathbf{Y}^{H}\right),
\end{aligned}
$$

where $\rho$ depends on $\mathbf{h}$ and $\boldsymbol{\Sigma}^{2}$ as $\rho=\mathbf{h}^{H} \boldsymbol{\Sigma}^{-2} \mathbf{h}$. We next optimize this expression under $\mathcal{H}_{0}$ and $\mathcal{H}_{1}$ to obtain the GLRT for each of the three models described in Section II. 
Copyright (c) 2016 IEEE. Personal use of this material is permitted. However, permission to use this material for any other purposes must be obtained from the IEEE by sending a request to pubs-permissions@ieee.org.

\section{A. Spatially white noise (Model 1)}

First, assume that the noise is i.i.d., that is, $\Sigma^{2}$ is a scaled version of the identity matrix (Section II, Model 1). The next result shows that the normalized data matrix,

$$
\overline{\mathbf{Y}} \doteq\left(\operatorname{tr}\left(\mathbf{Y} \mathbf{Y}^{H}\right)\right)^{-1 / 2} \mathbf{Y},
$$

is a sufficient statistic for GLR detection under this model.

Theorem 1 (GLRT under Model 1): The GLR test statistic under spatially white (sw) noise, i.e., when $\boldsymbol{\Sigma}^{2}=\sigma^{2} \mathbf{I}_{M}$, is

$$
\begin{aligned}
T_{\mathrm{sw}} & =\max _{\rho \geq 0} t_{\mathrm{sw}}(\rho), \\
t_{\mathrm{sw}}(\rho) & \doteq \frac{\left(1-\rho \lambda_{\max }\left(\overline{\mathbf{Y}} \mathbf{C}^{*}(\rho) \overline{\mathbf{Y}}^{H}\right)\right)^{-M N}}{\operatorname{det}\left(\mathbf{I}_{N}+\rho \mathbf{C}\right)} .
\end{aligned}
$$

Proof: See Appendix A-1.

The value of $\rho$ maximizing $t_{\mathrm{sw}}(\rho)$ cannot be expressed in closed form, except in certain cases. For white primary signals (those with uniform power spectrum $P_{s}(f)=1$ ), we may set $\mathbf{C}=\mathbf{I}_{N}$, so that (17) is equivalent to the test statistic $\lambda_{\max }\left(\overline{\mathbf{Y}} \overline{\mathbf{Y}}^{H}\right)=\frac{\lambda_{\max }\left(\mathbf{Y Y}^{H}\right)}{\operatorname{tr}\left(\mathbf{Y Y}^{H}\right)}$, and we recover the result in [4].

In the low-SNR regime, we may derive a GLR detector equivalent to (17) by considering the first coefficient of the Taylor series expansion of $\log t_{\mathrm{sw}}(\rho)$ as $\rho \rightarrow 0^{+}$. In Appendix A-2 it is shown that $T_{\mathrm{sw}}$ yields the following equivalent low-SNR closed-form test statistic,

$$
\begin{aligned}
\Lambda_{\mathrm{sw}}^{\mathrm{low}} & \doteq \max \left\{0, \lim _{\rho \rightarrow 0^{+}} \frac{1}{\rho} \log t_{\mathrm{sw}}(\rho)\right\} \\
& =\left[N M \lambda_{\max }\left(\overline{\mathbf{Y}} \mathbf{C}^{*} \overline{\mathbf{Y}}^{H}\right)-\operatorname{tr} \mathbf{C}^{*}\right]^{+} .
\end{aligned}
$$

Note that $\Lambda_{\mathrm{sw}}^{\text {low }}$ does not depend on $\rho$, as desired.

Let us now assume that the secondary user receives $P>1$ distinct signal components $\mathbf{s}_{i}$ with spatial signature $\mathbf{h}_{i}$ each. We may therefore express the data matrix $\mathbf{Y}$ as follows,

$$
\mathbf{Y}=\sum_{i=1}^{P} \mathbf{h}_{i} \mathbf{s}_{i}^{T}+\mathbf{N}=\mathbf{H S}^{T}+\mathbf{N},
$$

with $\mathbf{H} \doteq\left[\mathbf{h}_{1}, \cdots, \mathbf{h}_{P}\right], \mathbf{S} \doteq\left[\mathbf{s}_{1}, \cdots, \mathbf{s}_{P}\right]$ and noise matrix $\mathbf{N} \doteq\left[\mathbf{n}_{1}, \cdots, \mathbf{n}_{L}\right]$. We will refer to (21) as a signal model with spatial rank $P$ if the $M \times N$ matrix $\mathbf{H S}^{T}$ has row rank $P$ (or simply rank $P$, as, typically, we have that $N>>M$ ).

The low-SNR detector $\Lambda_{\mathrm{sw}}^{\text {low }}$ in (20) may be expressed as an optimization over a beamforming vector $\boldsymbol{w}$,

$$
\Lambda_{\mathrm{sw}}^{\text {low }}=\left[N M \max _{\boldsymbol{w}} \frac{\boldsymbol{w}^{H} \overline{\mathbf{Y}} \mathbf{C}^{*} \overline{\mathbf{Y}}^{H} \boldsymbol{w}}{\boldsymbol{w}^{H} \boldsymbol{w}}-\operatorname{tr} \mathbf{C}^{*}\right]^{+} .
$$

Thus, even under spatial rank $P>1$ for the primary signal, $\Lambda_{\mathrm{sw}}^{\text {low }}$ operates as a robust optimization criterion (i.e., without knowledge of $P$ ) by which the beamformer $\boldsymbol{w}$ is steered to collect the maximum energy from the spatial components of the normalized, time-filtered data matrix $\overline{\mathbf{Y}} \mathbf{C}^{* 1 / 2}$.

The low-SNR GRLT for $P=1, \Lambda_{\mathrm{sw}}^{\text {low }}$, appears as a correlation-matching multiantenna detector based on a statistical optimization criterion different from those in [7] (singleantenna non-GLR detectors for multiband signals) and [8] (single-antenna sub-Nyquist-sampling non-GLR detectors for sparse multiband signals). Both [7] and [8] explore alternative
Frobenius and geodetic distance measures between correlation matrices. Max-eigenvalue-type detectors based on energy combination as in (22) have also been derived in [36] for rank $P \geq 1$ signals in spatially i.i.d. noise. In particular, for $\mathbf{C}=\mathbf{I}_{N}$ and setting $L=1$ in [36], the BCED test in [36] and $\Lambda_{\mathrm{sw}}^{\text {low }}$ coincide, which attests to the applicability of $\Lambda_{\mathrm{sw}}^{\text {low }}$ even under spatial rank $P>1$. Simulations in Section VI-E will confirm this.

\section{B. Spatially uncorrelated noise (Model 2)}

When the noise is assumed independent across antennas, but not identically distributed, $\boldsymbol{\Sigma}^{2}$ is modeled as an arbitrary positive-definite diagonal matrix (Section II, Model 2). In this case, the derivation of the GLRT is more involved and cannot be reduced to a simple scalar optimization problem.

Let $\mathbf{D}^{2}$ denote the diagonal component of the sample correlation matrix $\frac{1}{N} \mathbf{Y} \mathbf{Y}^{H}$, i.e.,

$$
\mathbf{D}^{2} \doteq \frac{1}{N} \operatorname{diag}\left(\mathbf{Y} \mathbf{Y}^{H}\right) .
$$

Under $\mathcal{H}_{0}, \mathbf{h}=\mathbf{0}$ and the ML estimate of the noise covariance $\boldsymbol{\Sigma}^{2}$ is just $\mathbf{D}^{2}$. Substituting $\mathbf{h}=\mathbf{0}$ and $\boldsymbol{\Sigma}^{2}=\mathbf{D}^{2}$ in (15), we obtain the compressed log-likelihood

$$
\ell_{0} \doteq-M N \log \pi-N \log \operatorname{det} \mathbf{D}^{2}-M N .
$$

To find the ML estimates under $\mathcal{H}_{1}$, instead of the parameter space $\Omega=\{\boldsymbol{\Sigma}, \mathbf{h}\}$, we find it useful to define a new one $\Omega^{\prime}=\{\mathbf{T}, \overline{\boldsymbol{v}}, \rho\}$, with $\rho=\mathbf{h}^{H} \boldsymbol{\Sigma}^{-2} \mathbf{h}$ as before, and

$$
\begin{aligned}
& \mathbf{T} \doteq \mathbf{D} \boldsymbol{\Sigma}^{-1}, \\
& \overline{\boldsymbol{v}} \doteq \boldsymbol{v} /\|\boldsymbol{v}\|, \quad \boldsymbol{v} \doteq \mathbf{D} \boldsymbol{\Sigma}^{-2} \mathbf{h} .
\end{aligned}
$$

We easily check that $\|\boldsymbol{v}\|^{2}=\frac{\rho}{\overline{\boldsymbol{v}}^{H} \mathbf{T}^{-2} \overline{\boldsymbol{v}}}$. The mapping $\omega: \Omega \rightarrow$ $\Omega^{\prime}$ is bijective. Its inverse mapping $\omega^{-1}: \Omega^{\prime} \rightarrow \Omega$ is given by

$$
\boldsymbol{\Sigma}=\mathbf{D} \mathbf{T}^{-1} \quad, \quad \mathbf{h}=\sqrt{\frac{\rho}{\overline{\boldsymbol{v}}^{H} \mathbf{T}^{-2} \overline{\boldsymbol{v}}}} \mathbf{T}^{-2} \mathbf{D} \overline{\boldsymbol{v}} .
$$

Hence, it is equivalent to optimize the log-likelihood either over $\Omega$ or over $\Omega^{\prime}$. This fact will allow us to derive explicit expressions for the ML estimates under hypothesis $\mathcal{H}_{1}$.

Let us define the normalized data matrix,

$$
\tilde{\mathbf{Y}} \doteq \mathbf{D}^{-1} \mathbf{Y} \text {. }
$$

Using (27) and (28) we express (15) in the new space $\Omega^{\prime}$,

$$
\begin{aligned}
\log f & (\tilde{\mathbf{Y}} \mid \mathbf{T}, \overline{\boldsymbol{v}}, \rho)=-M N \log \pi \\
& +N \log \operatorname{det}\left(\mathbf{D}^{-2} \mathbf{T}^{2}\right)-\log \operatorname{det}\left(\mathbf{I}_{N}+\rho \mathbf{C}\right) \\
& -N \operatorname{tr}\left(\mathbf{T}^{2}\right)+\frac{\rho}{\overline{\boldsymbol{v}}^{H} \mathbf{T}^{-2} \overline{\boldsymbol{v}}} \operatorname{tr}\left(\overline{\boldsymbol{v}}^{H} \tilde{\mathbf{Y}} \mathbf{C}^{*}(\rho) \tilde{\mathbf{Y}}{ }^{H} \overline{\boldsymbol{v}}\right) .
\end{aligned}
$$

Using (24) and (29), we write the log-likelihood ratio as

$$
\begin{aligned}
& \begin{aligned}
& \Lambda(\tilde{\mathbf{Y}} \mid \mathbf{T}, \overline{\boldsymbol{v}}, \rho) \doteq \log f(\tilde{\mathbf{Y}} \mid \mathbf{T}, \overline{\boldsymbol{v}}, \rho)-\ell_{0} \\
&=-\log \operatorname{det}\left(\mathbf{I}_{N}+\rho \mathbf{C}\right) \\
&+N\left(\log \operatorname{det}\left(\mathbf{T}^{2}\right)-\operatorname{tr}\left(\mathbf{T}^{2}\right)+M+\frac{\beta(\overline{\boldsymbol{v}}, \rho)}{\overline{\boldsymbol{v}}^{H} \mathbf{T}^{-2} \overline{\boldsymbol{v}}}\right),
\end{aligned}
\end{aligned}
$$

where we defined

$$
\beta(\overline{\boldsymbol{v}}, \rho) \doteq \frac{\rho}{N} \overline{\boldsymbol{v}}^{H} \tilde{\mathbf{Y}} \mathbf{C}^{*}(\rho) \tilde{\mathbf{Y}}^{H} \overline{\boldsymbol{v}} .
$$


Copyright (c) 2016 IEEE. Personal use of this material is permitted. However, permission to use this material for any other purposes must be obtained from the IEEE by sending a request to pubs-permissions@ieee.org.

The next result provides the expression for the matrix $\mathbf{T}$ maximizing (31) in terms of $\overline{\boldsymbol{v}}$ and $\rho$.

Proposition 1: Let $\mathbf{T}(\overline{\boldsymbol{v}}, \rho)=\operatorname{diag}\left[t_{1}, \ldots, t_{M}\right]$ maximize (31) for $\overline{\boldsymbol{v}}$ and $\rho$ given. Then,

$$
t_{i}^{2}=\frac{1}{2}\left(1+\sqrt{1+\left|\bar{v}_{i}\right|^{2} q^{2}(\sqrt{\beta(\overline{\boldsymbol{v}}, \rho)})}\right),
$$

where $q(\tau)$ is the inverse of the function

$$
\tau(q) \doteq \sum_{i=1}^{M} \frac{\sqrt{1+q^{2}\left|\bar{v}_{i}\right|^{2}}-1}{q}=\sum_{i=1}^{M} \frac{q\left|\bar{v}_{i}\right|^{2}}{1+\sqrt{1+q^{2}\left|\bar{v}_{i}\right|^{2}}},
$$

for a given $\overline{\boldsymbol{v}}$. Moreover, for $\mathbf{T}=\mathbf{T}(\overline{\boldsymbol{v}}, \rho)$ it holds that

$$
\frac{\beta(\overline{\boldsymbol{v}}, \rho)}{\overline{\boldsymbol{v}}^{H} \mathbf{T}^{-2} \overline{\boldsymbol{v}}}=\operatorname{tr}\left(\mathbf{T}^{2}\right)-M
$$

Proof: See Appendix B-1.

Using (35) in (31) yields Theorem 2, which expresses the GLRT in terms of the compressed space $\Omega_{-}^{\prime}=\{\overline{\boldsymbol{v}}, \rho\}$.

Theorem 2 (GLRT under Model 2): The GLR test statistic under spatially uncorrelated $(\mathrm{su})$ noise, is given by the following optimization problem over the parameters $\{\overline{\boldsymbol{v}}, \rho\}$,

$$
\begin{aligned}
T_{\mathrm{su}} & =\max _{\rho \geq 0,\|\overline{\boldsymbol{v}}\|=1} t_{\mathrm{su}}(\overline{\boldsymbol{v}}, \rho), \\
t_{\mathrm{su}}(\overline{\boldsymbol{v}}, \rho) & \doteq \frac{\operatorname{det}^{N}\left[\mathbf{T}^{2}(\overline{\boldsymbol{v}}, \rho)\right]}{\operatorname{det}\left(\mathbf{I}_{N}+\rho \mathbf{C}\right)} .
\end{aligned}
$$

where $\mathbf{T}(\overline{\boldsymbol{v}}, \rho)$ depends on $\overline{\boldsymbol{v}}, \rho$ as stated in Proposition 1.

To compute $T_{\mathrm{su}}$ in (36), one must solve a joint optimization problem over $\overline{\boldsymbol{v}}$ and $\rho$. Since $t_{\mathrm{su}}(\overline{\boldsymbol{v}}, \rho)$ is non-convex, the optimization in (36) cannot be solved in closed form in general. Instead, Algorithm 1 proposes an iterative procedure to approximate (36), whereby we sequentially maximize (31) over $\{\overline{\boldsymbol{v}}, \rho\}$ with $\mathbf{T}$ fixed, and then over $\mathbf{T}$ with $\{\overline{\boldsymbol{v}}, \rho\}$ fixed. Since at each step the objective (31) cannot decrease, this alternating optimization scheme converges to a stationary point. While it is not guaranteed that the global maximum in (36) is attained, Algorithm 1 performs well in practice under several system models as will be shown in Section VI.

Analogously to the derivation of (20), Theorem 2 yields the following equivalent low-SNR test (see Appendix B-2),

$$
\Lambda_{\mathrm{su}}^{\text {low }} \doteq\left[\lambda_{\max }\left(\tilde{\mathbf{Y}} \mathbf{C}^{*} \tilde{\mathbf{Y}}^{H}\right)-\operatorname{tr} \mathbf{C}^{*}\right]^{+} .
$$

Setting $\mathbf{C}=\mathbf{I}_{N}$ in (38) and discarding irrelevant terms, the low-SNR detector $\lambda_{\max }\left(\tilde{\mathbf{Y}} \tilde{\mathbf{Y}}^{H}\right)=\lambda_{\max }\left(\mathbf{D}^{-1} \mathbf{Y} \mathbf{Y}^{H} \mathbf{D}^{-1}\right)$ derived in [6] for temporally white primary signals is recovered. Other particular scenarios in which the detector $T_{\mathrm{su}}$ can be simplified will be discussed in Section V. Under spatial rank $P>1$, analogous considerations to those after (22) apply.

\section{Spatially correlated noise (Model 3)}

When $\Sigma^{2}$ is an arbitrary full-rank positive definite matrix (Section II, Model 3), the corresponding GLRT is given in [12] and is included next for comparison purposes.

Theorem 3 (GLRT under Model 3): Let $\mathbf{Y}=\mathbf{U S V}^{H}$ be the reduced SVD of the data matrix Y. The GLR test statistic
Algorithm 1 Alternating optimization algorithm to approximate $T_{\mathrm{su}}$ in (36). Parameter $\delta$ indicates stop criterion.

1) Initialize $k \leftarrow 0, \mathbf{T}_{0} \leftarrow \mathbf{I}_{M}, T_{\mathrm{su}}^{(0)} \leftarrow 1$.

2) For $\mathbf{T}=\mathbf{T}_{k}$ maximize (31) with respect to $\{\overline{\boldsymbol{v}}, \rho\}$. We obtain

$$
\begin{gathered}
\rho_{k+1}=\arg \max _{\rho \geq 0}\left\{\rho \lambda_{\max }\left(\mathbf{T}_{k} \tilde{\mathbf{Y}} \mathbf{C}^{*}(\rho) \tilde{\mathbf{Y}}^{H} \mathbf{T}_{k}\right)\right. \\
\left.-\log \operatorname{det}\left(\mathbf{I}_{N}+\rho \mathbf{C}\right)\right\} .
\end{gathered}
$$

and, for $\boldsymbol{e}_{\max }$ the eigenvector associated to $\lambda_{\max }$ in $(\star)$

$$
\overline{\boldsymbol{v}}_{k+1}=\mathbf{T}_{k} \boldsymbol{e}_{\max } /\left\|\mathbf{T}_{k} \boldsymbol{e}_{\max }\right\| .
$$

3) Set $k \leftarrow k+1$; compute $\mathbf{T}_{k}=\mathbf{T}\left(\rho_{k}, \overline{\boldsymbol{v}}_{k}\right)$ (Propos. 1).

4) Update objective: $T_{\mathrm{su}}^{(k)}=\operatorname{det}^{N}\left(\mathbf{T}_{k}^{2}\right) / \operatorname{det}\left(\mathbf{I}_{N}+\rho_{k} \mathbf{C}\right)$. If $\frac{T_{\mathrm{su}}^{(k)}-T_{\mathrm{su}}^{(k-1)}}{T_{\mathrm{su}}^{(k)}} \leq \delta$, set $T_{\mathrm{su}} \leftarrow T_{\mathrm{su}}^{(k)}$ and finish.

Otherwise, go back to step 2).

under spatially correlated (sc) noise, i.e., when $\boldsymbol{\Sigma}^{2}$ is assumed arbitrary full-rank positive definite, is

$$
\begin{aligned}
T_{\mathrm{sc}} & =\max _{\rho \geq 0} t_{\mathrm{sc}}(\rho), \\
t_{\mathrm{sc}}(\rho) & \doteq \frac{\left(1-\rho \lambda_{\max }\left(\mathbf{V}^{H} \mathbf{C}^{*}(\rho) \mathbf{V}\right)\right)^{-N}}{\operatorname{det}\left(\mathbf{I}_{N}+\rho \mathbf{C}\right)} .
\end{aligned}
$$

In the low-SNR regime, Theorem 3 yields the following equivalent closed-form test statistic

$$
\Lambda_{\text {sc }}^{\text {low }}=\left[N \lambda_{\max }\left(\mathbf{V}^{H} \mathbf{C}^{*} \mathbf{V}\right)-\operatorname{tr}\left(\mathbf{C}^{*}\right)\right]^{+} .
$$

Note that, as was observed in [12], when $\mathbf{C}=\mathbf{I}_{N}$ the test statistic $\Lambda_{\mathrm{sc}}^{\text {low }}$ evaluates to a constant, independently of the semi-unitary data matrix $\mathbf{V}$. This is due to the unknown fullrank (unstructured) spatial correlation matrix assumed for the additive white noise. Hence, if the signal is also assumed white (i.e. $\mathbf{C}=\mathbf{I}_{N}$ ), it becomes indistinguishable from noise. Nevertheless, the detectors under Models 1 and 2, assume a spatial structure for the noise correlations and can operate even when a white spectrum for the primary signal is considered.

\section{Frequency domain interpretation}

Note that in the computation of the test statistics $T_{\mathrm{sw}}, T_{\mathrm{su}}$ and $T_{\mathrm{sc}}$, the data enters in the form $\mathbf{Z} \mathbf{C}^{*}(\rho) \mathbf{Z}^{H}$, where $\mathbf{Z}$ is an appropriately normalized $M \times N$ data matrix (either $\overline{\mathbf{Y}}$, $\tilde{\mathbf{Y}}$ or $\left.\mathbf{V}^{H}\right)$. Using the eigendecomposition $\mathbf{C}=\mathbf{U} \boldsymbol{\Lambda} \mathbf{U}^{H}$, it is seen that $\mathbf{C}(\rho)=\mathbf{U} \boldsymbol{\Lambda}(\rho) \mathbf{U}^{H}$, with $\boldsymbol{\Lambda}(\rho) \doteq \boldsymbol{\Lambda}\left(\mathbf{I}_{N}+\rho \boldsymbol{\Lambda}\right)^{-1}$. Writing $\mathbf{Z}$ row-wise as $\mathbf{Z}=\left[\begin{array}{lll}\mathbf{z}_{1} & \cdots & \mathbf{z}_{M}\end{array}\right]^{T}$, the $(i, j)$ element of $\mathbf{Z} \mathbf{C}^{*}(\rho) \mathbf{Z}^{H}$ is $\mathbf{z}_{j}^{H} \mathbf{C}(\rho) \mathbf{z}_{i}=\left(\mathbf{U}^{H} \mathbf{z}_{j}\right)^{H} \boldsymbol{\Lambda}(\rho)\left(\mathbf{U}^{H} \mathbf{z}_{i}\right)$. Asymptotically as $N \rightarrow \infty$, this can be seen as a spectrally weighted frequency-domain crosscorrelation between the outputs of the $i$-th and $j$-th normalized data streams, since $\mathbf{U}^{H} \mathbf{z}_{i}$ approaches the $N$-point DFT of $\mathbf{z}_{i}$. From (2), the spectral weights in $\boldsymbol{\Lambda}(\rho)$ approach the values of $\frac{P_{s}(f)}{1+\rho P_{s}(f)}$ at $f=\frac{k}{N}$. In the low SNR regime, these spectral weights are given by the sampled values of the PSD $P_{s}(f)$, as evidenced in the corresponding statistics $\Lambda_{\mathrm{sw}}, \Lambda_{\mathrm{su}}$ and $\Lambda_{\mathrm{sc}}$. 
Copyright (c) 2016 IEEE. Personal use of this material is permitted. However, permission to use this material for any other purposes must be obtained from the IEEE by sending a request to pubs-permissions@ieee.org.

With this view, it is clear that $\mathbf{Z} \mathbf{C}^{*}(\rho) \mathbf{Z}^{H}$ can be efficiently computed for large $N$ by fast Fourier transform (FFT) techniques. As discussed in [20], even for moderate values of $N$ the performance loss of this approach is negligible.

\section{Performance Analysis}

For a test statistic $T$ and threshold $\gamma$ in (9), the detection and false-alarm probabilities are respectively given by

$$
P_{\mathrm{D}}=\operatorname{Pr}\left\{T>\gamma \mid \mathcal{H}_{1}\right\} \quad, \quad P_{\mathrm{FA}}=\operatorname{Pr}\left\{T>\gamma \mid \mathcal{H}_{0}\right\}
$$

For the tests in Section III, $P_{\mathrm{D}}$ and $P_{\mathrm{FA}}$ in (42) can be approximated for sufficiently large $N$, using the asymptotic distribution of a GLR statistic $T_{\mathrm{GLR}}$ which, under suitable conditions [27], is given by

$$
2 \log T_{\mathrm{GLR}} \stackrel{\text { under }}{\sim} \mathcal{H}_{0} \chi_{r}^{2}, \quad 2 \log T_{\mathrm{GLR}} \stackrel{\text { under } \mathcal{H}_{1}}{\sim} \chi_{r}^{\prime 2}(\alpha),
$$

with $\chi_{r}^{2}$ and $\chi_{r}^{\prime 2}(\alpha)$ respectively the central and noncentral chi-square distributions with $r$ degrees of freedom, and noncentrality parameter $\alpha$. The number of degrees of freedom $r$ is given by the number of real parameters known under $\mathcal{H}_{0}$ and unknown under $\mathcal{H}_{1}$. In the three models presented, $r=2 M-1$ as the p.d.f. under $\mathcal{H}_{1}$ differs from that under $\mathcal{H}_{0}$ only in $\mathbf{h} \mathbf{h}^{H}$, i.e., $2 M$ real components of $\mathbf{h}$ minus one degree of freedom to account for the invariance to a complex rotation $e^{j \theta} \mathbf{h}$.

The noncentrality parameter $\alpha$ under each noise model can be computed as $\alpha=\mathbb{E}\left[2 \log T_{\mathrm{GLR}} \mid \mathcal{H}_{1}\right]$. Let " $\rightarrow$ " denote convergence as $N \rightarrow \infty$. The asymptotic values of $\alpha$ are derived in Appendix A-3 (Model 1), in Appendix B-3 (Model 2) and in [12, Eq. 74] (Model 3), and yield, respectively,

$$
\begin{aligned}
\alpha_{\mathrm{sw}} & \rightarrow 2 N \log \frac{\left(1+\frac{\rho}{M}\right)^{M}}{\operatorname{det}\left(\mathbf{I}_{N}+\rho \mathbf{C}\right)^{\frac{1}{N}}}, \\
\alpha_{\mathrm{su}} & \rightarrow 2 N \log \frac{\prod_{i=1}^{M}\left(1+\rho_{i}\right)}{\operatorname{det}\left(\mathbf{I}_{N}+\rho \mathbf{C}\right)^{\frac{1}{N}}}, \\
\alpha_{\mathrm{sc}} & \rightarrow 2 N \log \frac{1+\rho}{\operatorname{det}\left(\mathbf{I}_{N}+\rho \mathbf{C}\right)^{\frac{1}{N}}},
\end{aligned}
$$

where $\rho=\mathbf{h}^{H} \boldsymbol{\Sigma}^{-2} \mathbf{h}$ denotes the (true) overall SNR and $\rho_{i} \doteq$ $\frac{\left|h_{i}\right|^{2}}{\sigma_{i}^{2}}$ the SNR at the $i$-th antenna, $i=1, \ldots, M$.

If the spectrum of the signal of interest $s(t)$ does not contain spectral lines, an equivalent expression can be found in terms of its PSD $P_{s}(f)$. We define the spectral uniformity and spatial selectivity coefficients, respectively, as,

$$
\xi_{t} \doteq\left(\int_{0}^{1} P_{s}^{2}(f) \mathrm{d} f\right)^{-1}, \quad \xi_{s} \doteq \sum_{i=1}^{M}\left(\frac{\rho_{i}}{\rho}\right)^{2}
$$

with $0<\xi_{t} \leq 1$ (maximum achieved for a white spectrum $P_{s}(f)=1$ ) and $\frac{1}{M} \leq \xi_{s} \leq 1$ (minimum achieved for a uniform SNR profile $\rho_{1 \leq i \leq M}=\frac{\rho}{M}$, and maximum for $\left.\rho_{m}=\rho, \rho_{i \neq m}=0\right)$.

We denote convergence as $\rho \rightarrow 0^{+}$by " $\stackrel{0}{\longrightarrow}$ ". For small $x$ it follows that $\log (1+x)=x-\frac{1}{2} x^{2}+o\left(x^{2}\right)$. Then, using (2), from (44)-(46) we obtain

$$
\begin{aligned}
& \alpha_{\mathrm{sw}} \rightarrow 2 N \int_{0}^{1} \log \frac{\left(1+\frac{1}{M} \rho\right)^{M}}{1+\rho P_{s}(f)} \mathrm{d} f \stackrel{0}{\longrightarrow} \frac{\rho^{2} N}{\xi_{t}}\left(1-\frac{\xi_{t}}{M}\right), \\
& \alpha_{\mathrm{su}} \rightarrow 2 N \int_{0}^{1} \log \frac{\prod_{i=1}^{M}\left(1+\rho_{i}\right)}{1+\rho P_{s}(f)} \mathrm{d} f \stackrel{0}{\longrightarrow} \frac{\rho^{2} N}{\xi_{t}}\left(1-\xi_{t} \xi_{s}\right), \\
& \alpha_{\mathrm{sc}} \rightarrow 2 N \int_{0}^{1} \log \frac{1+\rho}{1+\rho P_{s}(f)} \mathrm{d} f \stackrel{0}{\longrightarrow} \frac{\rho^{2} N}{\xi_{t}}\left(1-\xi_{t}\right),
\end{aligned}
$$

where we note the trade-off between $\rho$ and $N$ in terms of the common factor $\rho^{2} N$. Then, from the convexity of $\log (\cdot)$ and Jensen's inequality,

$$
\alpha_{\mathrm{sw}} \geq \alpha_{\mathrm{su}} \geq \alpha_{\mathrm{sc}}(M>1) \quad, \quad \alpha_{\mathrm{sw}}=\alpha_{\mathrm{su}}=\alpha_{\mathrm{sc}}(M=1) .
$$

This ordering appeals to intuition as, for the same $P_{s}(f)$ and $\rho$, tests under Models 1 to 3 progressively need to estimate more parameters from the same amount of data. For $M=1$, the spatial correlation structure under all three noise models is identical, and the performance of the three detectors coincide.

For a white spectrum $P_{s}(f)=1$, i.e. $\xi_{t}=1$, the detector $T_{\mathrm{sc}}$ under spatially correlated noise (Model 3 ) collapses, and $\alpha_{\mathrm{sc}}=0$. However, for $M>1$, detectors $T_{\mathrm{sw}}$ and $T_{\mathrm{su}}$ do not. This is due to the spatial correlation structure of the noise, which is distinguishable from that of the signal of interest under Models 1 and 2.

While (43) is an asymptotic result, it approximates well the true statistics of the tests even for moderate sample sizes. Fig. 1 compares the analytical and empirical distributions of the three detectors ${ }^{1}$ for a Gaussian primary signal with binary PSD occupying half of the bandwidth. We observe a good match between the analytical and empirical distributions for a sample size as small as $N=128$.

\section{Some Particular Cases}

In some scenarios, the GLR tests derived in Section III adopt a simpler form. In this section we particularize these tests (i) for the single antenna case, (ii) under different per-antenna SNR profiles, and (iii) for specific covariance matrices of the primary signal. Table I lists the GLR detectors under the three noise models, both for the all-SNR and low-SNR regimes. The parameters of the detectors obtained under a white or binary PSD (henceforth binary PSD) are summarized in Table II.

\section{A. Single Antenna}

The performance of the GLR detectors under Models 1, 2 and 3, as shown in Section IV, coincide for $M=1$. Particularizing these detectors using $\overline{\mathbf{Y}}=\tilde{\mathbf{Y}} / N=\mathbf{V}^{H}=\mathbf{y}^{T} / \sqrt{\mathbf{y}^{H} \mathbf{y}}$ shows that they are indeed the same. In particular, defining $\overline{\mathbf{y}} \doteq \mathbf{y} / \sqrt{\mathbf{y}^{H} \mathbf{y}}$, the single antenna GLRT becomes,

$$
\begin{aligned}
T_{1} & \doteq \max _{\rho \geq 0} t_{1}(\rho), \\
t_{1}(\rho) & \doteq \frac{\left(1-\rho \overline{\mathbf{y}}^{T} \mathbf{C}^{*}(\rho) \overline{\mathbf{y}}^{*}\right)^{-N}}{\operatorname{det}\left(\mathbf{I}_{N}+\rho \mathbf{C}\right)}=\frac{\left(\overline{\mathbf{y}}^{H}\left(\mathbf{I}_{N}+\rho \mathbf{C}\right)^{-1} \overline{\mathbf{y}}\right)^{-N}}{\operatorname{det}\left(\mathbf{I}_{N}+\rho \mathbf{C}\right)} .
\end{aligned}
$$

\footnotetext{
${ }^{1}$ For implementation details, see Section VI.
} 
Copyright (c) 2016 IEEE. Personal use of this material is permitted. However, permission to use this material for any other purposes must be obtained from the IEEE by sending a request to pubs-permissions@ieee.org.
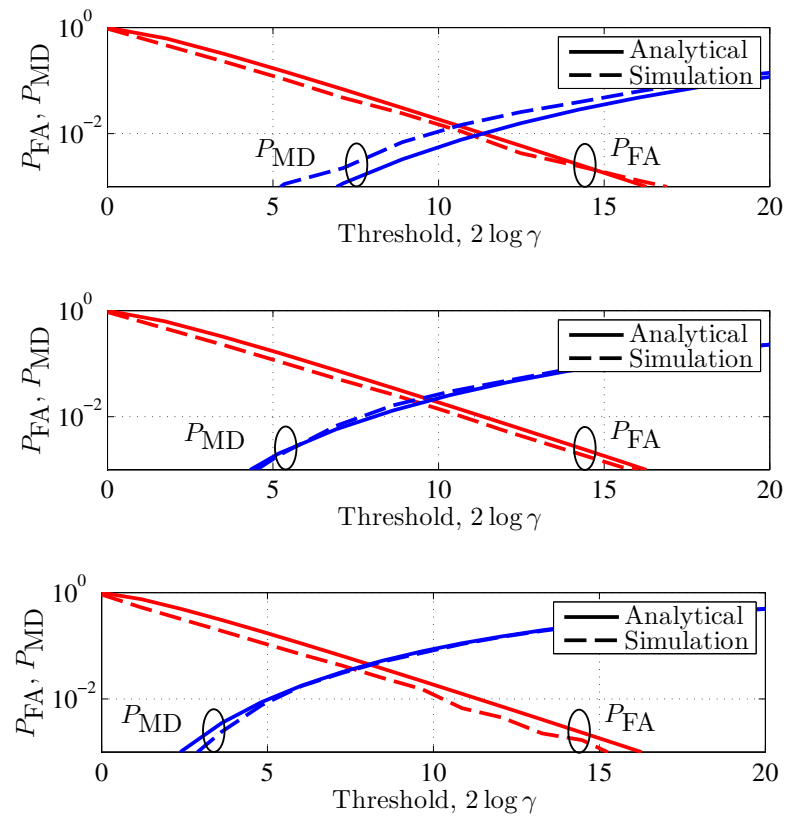

Fig. 1. Asymptotic (solid) and empirical (dashed) probabilities of false-alarm $P_{\mathrm{FA}}$ and misdetection $P_{\mathrm{MD}}=1-P_{\mathrm{D}}$ vs. threshold for (a) $T_{\mathrm{sw}}$, (b) $T_{\mathrm{su}}$ and (c) $T_{\mathrm{sc}}$. Gaussian primary signal with binary PSD, spatially i.i.d. noise, $M=2, N=256, \rho=-4.5 \mathrm{~dB}$. The distribution of $2 \log T_{\mathrm{su}}$ under $\mathcal{H}_{1}$ has been averaged over channel realizations.

In the low SNR regime (52)-(53) yields

$$
\Lambda_{1}^{\text {low }}=\left[N \frac{\mathbf{y}^{H} \mathbf{C y}}{\mathbf{y}^{H} \mathbf{y}}-\operatorname{tr}(\mathbf{C})\right]^{+} .
$$

\section{B. Particular per-antenna SNR profiles}

Consider the spatially uncorrelated noise model (Section II, Model 2). We shall particularize the detectors $T_{\text {su }}$ and its low-SNR equivalent form $\Lambda_{\mathrm{su}}^{\text {low }}$ for different per-antenna SNR profiles. We proceed first with an auxiliary result.

Consider the per-antenna SNR vector $\boldsymbol{\rho} \doteq\left[\rho_{1}, \ldots, \rho_{M}\right]^{T}$, with $\rho_{i}=\frac{\left|h_{i}\right|^{2}}{\sigma_{i}^{2}}$. Using (27), it follows that

$$
\rho_{i}=\frac{\left|h_{i}\right|^{2}}{\sigma_{i}^{2}}=\rho \frac{t_{i}^{-2}\left|\bar{v}_{i}\right|^{2}}{\sum_{i=1}^{M} t_{i}^{-2}\left|\bar{v}_{i}\right|^{2}} .
$$

We seek to relate $\rho_{i}$ to $\left|\bar{v}_{i}\right|^{2}$ in terms of $\beta(\overline{\boldsymbol{v}}, \rho)$ only. The denominator in (55) can be obtained ${ }^{2}$ from (95) in Appendix B-1 as $\sum_{i=1}^{M} t_{i}^{-2}\left|\bar{v}_{i}\right|^{2}=2 \sqrt{\beta(\overline{\boldsymbol{v}}, \rho)} / q(\sqrt{\beta(\overline{\boldsymbol{v}}, \rho)})$. Additionally, from Proposition 1, we substitute $t_{i}^{2}$ in (33) into (55), which allows to relate $\rho_{i}$ to $\left|\bar{v}_{i}\right|^{2}$ as follows,

$$
\rho_{i}=\frac{q(\sqrt{\beta(\overline{\boldsymbol{v}}, \rho)})}{\sqrt{\beta(\overline{\boldsymbol{v}}, \rho)}} \frac{\rho\left|\bar{v}_{i}\right|^{2}}{1+\sqrt{1+\left|\bar{v}_{i}\right|^{2} q^{2}(\sqrt{\beta(\overline{\boldsymbol{v}}, \rho)})}} .
$$

We next study three particular scenarios in which $T_{\mathrm{su}}$ and $\Lambda_{\mathrm{su}}^{\text {low }}$ adopt a simple expression.

\footnotetext{
${ }^{2}$ We use the fact that the term $\phi$ in (95) is precisely the implicit function $q(\sqrt{\beta(\overline{\boldsymbol{v}}, \rho)})$, as established by (100) and Proposition 1 .
}

1) Single active antenna: Assume that the per-antenna SNR profile follows a delta distribution in which all but the $i$-th antenna have zero SNR, with $i$ unknown, i.e., $\boldsymbol{\rho}=\rho \boldsymbol{\delta}_{i}$ with $\delta_{i}$ the $i$-th pinning vector. Our purpose herein is to show that this particular SNR distribution model, although unlikely in a scenario of co-located antennas, is naturally associated with a GLR detector based on selection combining, a well-known strategy in multiantenna signal processing.

Substituting $\boldsymbol{\rho}=\rho \boldsymbol{\delta}_{i}$ in (56), the search over $\overline{\boldsymbol{v}}$ can be constrained to vectors of the form $\overline{\boldsymbol{v}}=e^{j \theta} \boldsymbol{\delta}_{i}$ where $\theta$ is an arbitrary phase term. Setting $\overline{\boldsymbol{v}}=e^{j \theta} \boldsymbol{\delta}_{i}$ in (34) we obtain $q(\tau)=\frac{2 \tau}{1-\tau^{2}}$. Then, after some algebra, Proposition 1 yields,

$$
t_{k}^{2}= \begin{cases}\left(1-\frac{\rho}{N} \tilde{\mathbf{y}}_{i}^{T} \mathbf{C}^{*}(\rho) \tilde{\mathbf{y}}_{i}^{*}\right)^{-1}, & k=i, \\ 1, & k \neq i,\end{cases}
$$

for $k=1, \ldots, M$, and with $\tilde{\mathbf{y}}_{i}^{T} \doteq \boldsymbol{\delta}_{i}^{T} \tilde{\mathbf{Y}}$ the $i$-th row of $\tilde{\mathbf{Y}}$.

Substituting (57) in (36)-(37), optimizing over vectors of the form $\overline{\boldsymbol{v}}=e^{j \theta} \boldsymbol{\delta}_{i}$, we obtain the detector

$$
\begin{aligned}
T_{\mathrm{su}-\mathrm{sc}} & \doteq \max _{1 \leq i \leq M} \max _{\rho \geq 0} t_{\mathrm{su}}^{(i)}(\rho), \\
t_{\mathrm{su}}^{(i)}(\rho) & \doteq \frac{\left(1-\frac{\rho}{N} \tilde{\mathbf{y}}_{i}^{T} \mathbf{C}^{*}(\rho) \tilde{\mathbf{y}}_{i}^{*}\right)^{-N}}{\operatorname{det}\left(\mathbf{I}_{N}+\rho \mathbf{C}\right)} \\
& =\frac{\left(\frac{1}{N} \tilde{\mathbf{y}}_{i}^{H}\left(\mathbf{I}_{N}+\rho \mathbf{C}\right)^{-1} \tilde{\mathbf{y}}_{i}\right)^{-N}}{\operatorname{det}\left(\mathbf{I}_{N}+\rho \mathbf{C}\right)} .
\end{aligned}
$$

That is, the detector simply operates as a selection combining (sc) scheme. At low-SNR, i.e., for $\rho \rightarrow 0^{+}, T_{\text {su-sc }}$ yields

$$
\Lambda_{\mathrm{su}-\mathrm{sc}}^{\mathrm{low}}=\left[\max _{1 \leq i \leq M} \tilde{\mathbf{y}}_{i}^{H} \mathbf{C} \tilde{\mathbf{y}}_{i}-\operatorname{tr} \mathbf{C}\right]^{+} .
$$

We compare $\Lambda_{\mathrm{su}-\mathrm{sc}}^{\text {low }}$ against the selection combining detector in [11, Sec. IV-B, Eq. (24)], which was derived in the low SNR regime for i.i.d. noise with known variance, $\max _{1 \leq i \leq M} \overline{\mathbf{y}}_{i}^{H} \mathbf{C} \overline{\mathbf{y}}_{i}$, where $\overline{\mathbf{y}}_{i}^{T}=\boldsymbol{\delta}_{i}^{T} \mathbf{Y}$ denotes the $i$-th row of $\mathbf{Y}$. Since $\tilde{\mathbf{y}}_{i}=N \overline{\mathbf{y}}_{i} /\left\|\overline{\mathbf{y}}_{i}\right\|$, we can see that, compared with the detector from [11], $\Lambda_{\text {su-sc }}^{\text {low }}$ includes a normalization term due to the lack of knowledge about the noise variances.

2) Uniform per-antenna SNR profile: Assume now the same SNR for all antennas: $\rho_{i}=\frac{\rho}{M}$. We will show that this particular SNR distribution model is naturally associated with a GLR detector based on equal gain combining, another well-known strategy in multiantenna signal processing. This may approximately occur, for instance, when receiving distant sources or under Rice fading, with similar per-antenna signal (and noise) powers. Another approach to this scenario is also pursued in the following subsection.

Let us define the equal gain combining (egc) vectors $\psi \doteq$ $\frac{1}{\sqrt{M}}\left[e^{j \theta_{1}}, \cdots, e^{j \theta_{M}}\right]^{T}, 0 \leq \theta_{i} \leq 2 \pi$ for arbitrary phases $\theta_{i}$, $i=1, \ldots, M$. From (56) we have that the constraint $\rho_{i}=\frac{\rho}{M}$ implies $\left|\bar{v}_{i}\right|^{2}=\frac{1}{M}, i=1, \ldots, M$. Hence, the optimization over $\overline{\boldsymbol{v}}$ can be replaced by an optimization over the set of egc vectors $\boldsymbol{\psi}$. For $\overline{\boldsymbol{v}}=\boldsymbol{\psi}$, after some algebra, (34) yields $q(\tau)=\frac{2 \tau}{1-\tau^{2} / M}$ and (33) becomes

$$
t_{i}^{2}=\left(1-\frac{1}{M} \beta(\rho, \boldsymbol{\psi})\right)^{-1} .
$$


Copyright (c) 2016 IEEE. Personal use of this material is permitted. However, permission to use this material for any other purposes must be obtained from the IEEE by sending a request to pubs-permissions@ieee.org.

Substituting (62) in (36)-(37), and using (32), we obtain the GLRT for a uniform per-antenna SNR profile,

$$
\begin{aligned}
T_{\text {su-egc }} & =\max _{\rho \geq 0, \boldsymbol{\psi}} t_{\text {su-egc }}(\rho, \boldsymbol{\psi}), \\
t_{\text {su-egc }}(\rho, \boldsymbol{\psi}) & \doteq \frac{\left(1-\frac{\rho}{N M} \boldsymbol{\psi}^{H} \tilde{\mathbf{Y}} \mathbf{C}^{*}(\rho) \tilde{\mathbf{Y}}^{H} \boldsymbol{\psi}\right)^{-N M}}{\operatorname{det}\left(\mathbf{I}_{N}+\rho \mathbf{C}\right)} .
\end{aligned}
$$

In the low SNR regime as $\rho \rightarrow 0^{+}, T_{\text {su-egc }}$ yields

$$
\Lambda_{\text {su-egc }}^{\text {low }} \doteq\left[\max _{\psi}\left(\psi^{H} \tilde{\mathbf{Y}} \mathbf{C}^{*} \tilde{\mathbf{Y}}^{H} \boldsymbol{\psi}\right)-\operatorname{tr} \mathbf{C}\right]^{+}
$$

We compare this detector against the EGC detector in [11, Sec. IV-C], $\max _{\psi} \boldsymbol{\psi}^{H} \mathbf{Y} \mathbf{C}^{*} \mathbf{Y}^{H} \boldsymbol{\psi}$, derived in the low SNR regime for i.i.d. noise with known variance. Again, noise uncertainty translates into a normalization term via $\tilde{\mathbf{Y}}=\mathbf{D}^{-1} \mathbf{Y}$ in (65).

3) Similar SNR profile: In order to improve the SNR model for the scenario contemplated in the previous subsection, we consider now a small perturbation of the uniform SNR profile $\rho_{i}=\frac{\rho}{M}$. In particular, set $\left|\bar{v}_{i}\right|^{2}=\frac{1}{M}+\nu_{i}$, with $\nu_{i}$ small perturbations such that $\sum_{i=1}^{M} \nu_{i}=0$.

From (34), and using that for small $|x|$ it holds that $\sqrt{1+x} \simeq 1+\frac{x}{2}$, we obtain the following approximation

$$
\begin{aligned}
\tau & =\frac{1}{q} \sum_{i=1}^{M}\left(\sqrt{1+q^{2}\left(\frac{1}{M}+\nu_{i}\right)}-1\right) \\
& \simeq \frac{1}{q} \sum_{i=1}^{M}\left(\sqrt{1+\frac{1}{M} q^{2}}-1\right)+\frac{1}{2} \sum_{i=1}^{M} \frac{\nu_{i} q}{\sqrt{1+q^{2} / M}} .
\end{aligned}
$$

Thus, as $\sum_{i=1}^{M} \nu_{i}=0$, the second term in (67) is zero and the value of $q(\tau)$ derived for a uniform per-antenna SNR profile approximates the true $q(\tau)$ up to second-order. This makes the test $T_{\text {su-egc }}$ in (63) robust to small deviations from a uniform SNR profile. Then, alternatively to the optimization in (63), we take $\boldsymbol{\psi}=\overline{\boldsymbol{v}}$, with $\overline{\boldsymbol{v}}$ unconstrained, and we obtain the similar gain combiner (sgc) detector

$$
\begin{aligned}
T_{\mathrm{su}-\mathrm{sgc}} & =\max _{\rho \geq 0} t_{\mathrm{su}-\mathrm{sgc}}(\rho), \\
t_{\mathrm{su}-\mathrm{sgc}}(\rho) & \doteq \frac{\left(1-\frac{\rho}{N M} \lambda_{\max }\left(\tilde{\mathbf{Y}} \mathbf{C}^{*}(\rho) \tilde{\mathbf{Y}}^{H}\right)\right)^{-N M}}{\operatorname{det}\left(\mathbf{I}_{N}+\rho \mathbf{C}\right)} .
\end{aligned}
$$

This detector has the same form as that in Theorem 1, derived for spatially white noise (Model 1). Note however the different normalization terms, $\overline{\mathbf{Y}}=\mathbf{Y} / \sqrt{\operatorname{tr}\left(\mathbf{Y} \mathbf{Y}^{H}\right)}$ under Model 1 and $\tilde{\mathbf{Y}}=\mathbf{D}^{-1} \mathbf{Y}$ under Model 2. The low-SNR detector corresponding to (69) is precisely $\Lambda_{\mathrm{su}-\mathrm{sgc}}^{\mathrm{low}}=\Lambda_{\mathrm{su}}^{\mathrm{low}}$ in (38).

\section{Binary power spectrum}

Consider a primary signal with binary power spectrum (bs), i.e., with power spectrum $P_{s}(f) \in\{0, S\}$ where $S>0$ does not depend on $f$. This is a model of interest for detectors that only exploit bandwidth occupancy information, as the template spectrum $P_{s}(f)$ evaluates to $S$ in those frequencies containing signal components and to 0 in those where only noise is to be expected. Although such detectors do not fully exploit the power spectral shape, they can be easily derived under the current framework. Moreover, we will show that when the

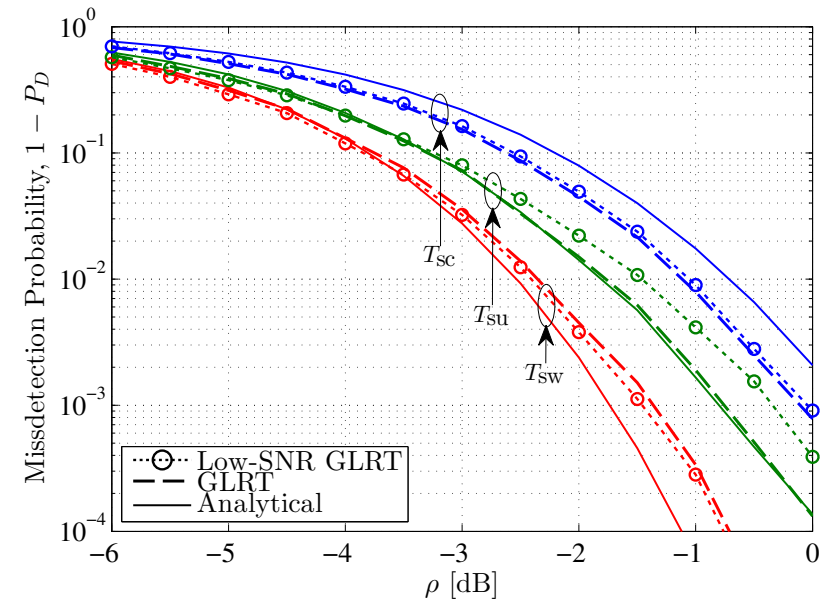

Fig. 2. Gaussian primary signal with binary PSD and spatially i.i.d. noise (Model 1), $M=2, N=128, P_{\mathrm{FA}}=0.01$.

primary signal fits this model, the corresponding low-SNR detector is equivalent to the all-SNR detector.

Now, using (2), it follows for large $N$ that the correlation matrix $\mathbf{C}$ can be modeled as $\mathbf{C}=\mu \mathbf{U}_{c} \mathbf{U}_{c}^{H}$, where $\mu=N / r$ is a normalization constant and $\mathbf{U}_{c} \in \mathbb{C}^{N \times r}$ is semi-unitary, i.e, $\mathbf{U}_{c}^{H} \mathbf{U}_{c}=\mathbf{I}_{r}$, with $r$ the rank of $\mathbf{C}$. Then,

$$
\rho \mathbf{C}(\rho)=\frac{\rho \mu}{1+\rho \mu} \mathbf{U}_{c} \mathbf{U}_{c}^{H}, \operatorname{det}\left(\mathbf{I}_{N}+\rho \mathbf{C}\right)=(1+\rho \mu)^{r} .
$$

Using (70), except for the general detector under spatially uncorrelated noise model (Model 2), the detectors in Table I can be written in the following form,

$$
T_{\mathrm{bs}}=\max _{\rho \geq 0}\left\{(1+\rho \mu)^{-r}\left(1-\frac{\rho \mu}{1+\rho \mu} b\right)^{-L}\right\},
$$

where the parameters $b$ and $L$ are specified in Table II for the different noise models. As shown in Appendix C, this optimization can be solved in closed form, yielding

$$
T_{\mathrm{bs}}=\max \left\{1, \frac{(r / L)^{r}(1-r / L)^{L-r}}{b^{r}(1-b)^{L-r}}\right\}
$$

where $\rho_{\star}=\frac{1}{\mu r} \max \left\{0, \frac{L b-r}{1-b}\right\}$ attains the maximum in (71). In the low SNR regime, for the detectors in Table II, we get

$$
\Lambda_{\mathrm{bs}}^{\mathrm{low}}=\max \{0, L b-r\} .
$$

As (72) is non-decreasing in $b \in\left[\frac{r}{L}, 1\right)$, the tests (72) and (73) are both equivalent. Thus, for the cases in Table II, the detectors developed under a low-SNR approximation are in fact equivalent to the all-SNR tests for binary power spectrum. This is not the case though for detector $T_{\mathrm{su}}$ and its low-SNR approximation $\Lambda_{\mathrm{su}}^{\text {low }}$, or for a general PSD (see Section VI).

\section{Simulation Results}

We evaluate the performance of the proposed detectors under noise Models 1 and 2. The detector $T_{\mathrm{su}}$ is implemented with Algorithm 1. For all the detectors, the optimization over $\rho$ employs Newton-Raphson's method, with starting point $\rho=0$. 
Copyright (c) 2016 IEEE. Personal use of this material is permitted. However, permission to use this material for any other purposes must be obtained from the IEEE by sending a request to pubs-permissions@ieee.org.

\begin{tabular}{|c|c|c|c|}
\hline Spatial Noise Model & Data & all-SNR GLRT $(T)$ & low-SNR GLRT $\left(a_{1}\right)$ \\
\hline (1) spatially white & $\overline{\mathbf{Y}}=\frac{\mathbf{Y}}{\sqrt{\operatorname{tr}\left(\mathbf{Y} \mathbf{Y}^{H}\right)}}$ & $\max _{\rho \geq 0} \frac{\left(1-\rho \lambda_{\max }\left(\overline{\mathbf{Y}} \mathbf{C}^{*}(\rho) \overline{\mathbf{Y}}^{H}\right)\right)^{-N M}}{\operatorname{det}\left(\mathbf{I}_{N}+\rho \mathbf{C}\right)}$ & $N M \lambda_{\max }\left(\overline{\mathbf{Y}} \mathbf{C}^{*} \overline{\mathbf{Y}}^{H}\right)-\operatorname{tr} \mathbf{C}$ \\
\hline (2) spatially uncorrelated & $\tilde{\mathbf{Y}}=\mathbf{D}^{-1} \mathbf{Y}$ & $\max _{\rho \geq 0, \overline{\boldsymbol{v}}} \frac{\left(\Pi_{k=1}^{M} t_{i}^{2}(\overline{\boldsymbol{v}}, \rho)\right)^{N}}{\operatorname{det}\left(\mathbf{I}_{N}+\rho \mathbf{C}\right)}$ & $\lambda_{\max }\left(\tilde{\mathbf{Y}} \mathbf{C}^{*} \tilde{\mathbf{Y}}^{H}\right)-\operatorname{tr} \mathbf{C}$ \\
\hline - Selection Combiner, $\xi_{s}=1$ & $\tilde{\mathbf{y}}_{i}^{T}=\operatorname{row}_{i}(\tilde{\mathbf{Y}})$ & $\max _{1 \leq i \leq M, \rho \geq 0} \frac{\left(1-\frac{\rho}{N} \tilde{\mathbf{y}}_{i}^{T} \mathbf{C}^{*}(\rho) \tilde{\mathbf{y}}_{i}\right)^{-N}}{\operatorname{det}\left(\mathbf{I}_{N}+\rho \mathbf{C}\right)}$ & $\max _{1 \leq i \leq M}\left(\tilde{\mathbf{y}}_{i}^{T} \mathbf{C}^{*} \tilde{\mathbf{y}}_{i}^{*}\right)-\operatorname{tr} \mathbf{C}$ \\
\hline - Eq. Gain Combiner, $\xi_{s}=\frac{1}{M}$ & & $\max _{\rho \geq 0, \boldsymbol{\psi}} \frac{\left(1-\frac{\rho}{N M} \boldsymbol{\psi}^{H} \tilde{\mathbf{Y}} \mathbf{C}^{*}(\rho) \tilde{\mathbf{Y}}^{H} \boldsymbol{\psi}\right)^{-N M}}{\operatorname{det}\left(\mathbf{I}_{N}+\rho \mathbf{C}\right)}$ & $\max _{\boldsymbol{\psi}}\left(\boldsymbol{\psi}^{H} \tilde{\mathbf{Y}} \mathbf{C}^{*} \tilde{\mathbf{Y}}^{H} \boldsymbol{\psi}\right)-\operatorname{tr} \mathbf{C}$ \\
\hline - Sim. Gain Combiner, $\xi_{s} \gtrsim \frac{1}{M}$ & & $\max _{\rho \geq 0} \frac{\left(1-\frac{\rho}{N M} \lambda_{\max }\left(\tilde{\mathbf{Y}} \mathbf{C}^{*}(\rho) \tilde{\mathbf{Y}}^{H}\right)\right)^{-N M}}{\operatorname{det}\left(\mathbf{I}_{N}+\rho \mathbf{C}\right)}$ & $\lambda_{\max }\left(\tilde{\mathbf{Y}} \mathbf{C}^{*} \tilde{\mathbf{Y}}^{H}\right)-\operatorname{tr} \mathbf{C}$ \\
\hline (3) spatially correlated, $\mathbf{C} \neq \mathbf{I}$ & $\mathbf{Y}=\mathbf{U S V}^{H}$ & $\max _{\rho \geq 0} \frac{\left(1-\rho \lambda_{\max }\left(\mathbf{V}^{H} \mathbf{C}^{*}(\rho) \mathbf{V}\right)\right)^{-N}}{\operatorname{det}\left(\mathbf{I}_{N}+\rho \mathbf{C}\right)}$ & $N \lambda_{\max }\left(\mathbf{V}^{H} \mathbf{C}^{*} \mathbf{V}\right)-\operatorname{tr} \mathbf{C}$ \\
\hline
\end{tabular}

TABLE I

SUMMARY OF GLR DETECTORS UNDER THE THREE NOISE MODELS, WITH $\Lambda=\left[a_{1}\right]^{+}$THE LOW-SNR DETECTOR.

\begin{tabular}{|l|c|c|}
\hline Spatial Noise Model & Data & All-SNR and low-SNR detectors (72) and (73) with parameters: \\
\hline (1) spatially white & $\overline{\mathbf{Y}}_{c}=\overline{\mathbf{Y}} \mathbf{U}_{c}$ & $b=\lambda_{\max }\left(\overline{\mathbf{Y}}_{c} \overline{\mathbf{Y}}_{c}^{H}\right), L=N M$ \\
\hline (2) spatially uncorrelated & $\tilde{\mathbf{Y}}_{c}=\tilde{\mathbf{Y}} \mathbf{U}_{c}$ & [no explicit form available] \\
$\bullet$ Selection Combiner, $\xi_{s}=1$ & $\tilde{\mathbf{y}}_{c, i}^{T}=\operatorname{row}_{i}\left(\tilde{\mathbf{Y}}_{c}\right)$ & $b=\max _{1 \leq i \leq M}\left(\frac{1}{N} \tilde{\mathbf{y}}_{c, i}^{H} \tilde{\mathbf{y}}_{c, i}\right), L=N$ \\
$\bullet$ Eq. Gain Combiner, $\xi_{s}=\frac{1}{M}$ & & $b=\frac{1}{N M} \max _{\boldsymbol{\psi}}\left(\boldsymbol{\psi}^{H} \tilde{\mathbf{Y}}_{c} \tilde{\mathbf{Y}}_{c}^{H} \boldsymbol{\psi}\right), L=N M$ \\
$\bullet$ Sim. Gain Combiner, $\xi_{s} \gtrsim \frac{1}{M}$ & & $b=\frac{1}{N M} \lambda_{\max }\left(\tilde{\mathbf{Y}}_{c} \tilde{\mathbf{Y}}_{c}^{H}\right), L=N M$ \\
\hline (3) spatially correlated & $\mathbf{V}_{c}=\mathbf{U}_{c}^{H} \mathbf{V}$ & $b=\lambda_{\max }\left(\mathbf{V}_{c} \mathbf{V}_{c}^{H}\right), L=N$ \\
\hline
\end{tabular}

SUMMARY OF GLR DETECTORS IN TABLE I IN EXPLICIT FORM FOR A BINARY PSD.

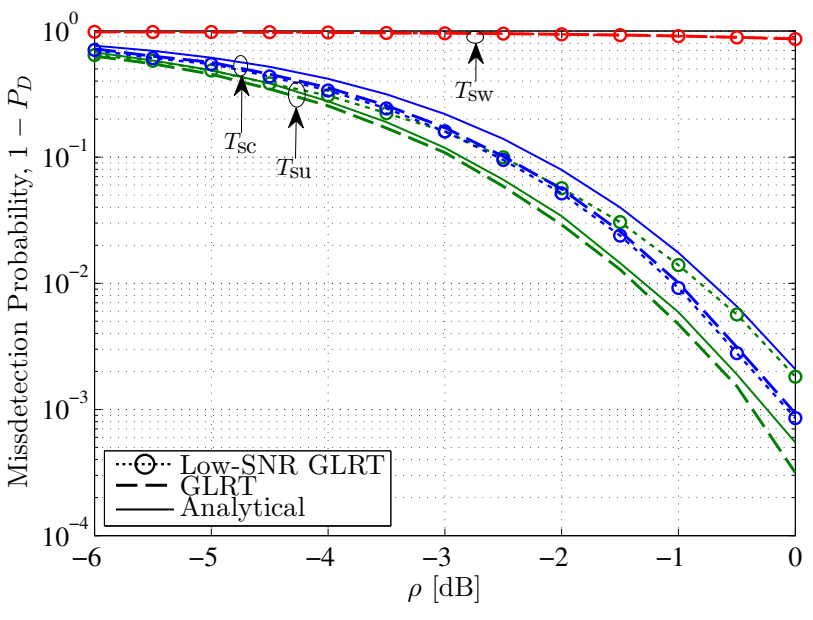

Fig. 3. Gaussian primary signal with binary PSD and spatially uncorrelated noise (Model 2), $M=2, N=128, P_{\mathrm{FA}}=0.01$.

\section{A. Calibrated vs. uncalibrated receivers}

First, we evaluate the performance of detectors $T_{\mathrm{sw}}, T_{\mathrm{su}}$ and $T_{\mathrm{sc}}$, and their low-SNR approximations $\Lambda_{\mathrm{sw}}^{\text {low }}, \Lambda_{\mathrm{su}}^{\text {low }}$ and $\Lambda_{\mathrm{sc}}^{\text {low }}$ with respect to the SNR. We also compare these results with those predicted by the approximate statistical distributions of the tests presented in Section IV. Consider a Gaussian primary signal with binary PSD occupying half of the bandwidth. For this particular signal, the spectral uniformity coefficient in (47) is $\xi_{t}=0.5$. We fix $M=2$ antennas and $N=128$ samples.

We perform Monte Carlo simulation with $10^{5}$ runs per point. In order to establish a fair comparison between the performance of the three detectors, and in accordance with the performance results in section IV, at each run the channel coefficients are drawn from a Rayleigh distribution and normalized to have a constant SNR $\rho=\mathbf{h}^{H} \boldsymbol{\Sigma}^{-2} \mathbf{h}$. Thresholds are chosen to yield $P_{\mathrm{FA}}=0.01$.

Fig. 2 shows the misdetection probability $1-P_{\mathrm{D}}$ versus the SNR $\rho$ when we fix $\Sigma^{2}=\mathbf{I}_{2}$ (noise Model 1). The detector $T_{\mathrm{sw}}$, derived precisely for $\boldsymbol{\Sigma}^{2}=\sigma^{2} \mathbf{I}_{2}$, performs best. On the other hand, detectors $T_{\mathrm{su}}$ and $T_{\mathrm{sc}}$, derived under more general models, incur a performance loss. This agrees with the asymptotic performance of the three detectors in Section IV.

Fig. 3 shows the results for the same scenario as in Fig. 2, except for the underlying noise model, which now is Model 2. The diagonal elements of $\boldsymbol{\Sigma}^{2}$ are drawn in each run from a $\chi_{1}^{2}$ distribution. Note that while the performance of $T_{\mathrm{su}}$ and $T_{\mathrm{sc}}$ is similar to that in Fig. 2, $T_{\mathrm{sw}}$ degrades considerably due to the noise model mismatch. The analytical curve of $T_{\mathrm{sw}}$ is not shown in Fig. 3, as it does not apply under a mismatched model. This shows the sensitivity of this detector to calibration 
Copyright (c) 2016 IEEE. Personal use of this material is permitted. However, permission to use this material for any other purposes must be obtained from the IEEE by sending a request to pubs-permissions@ieee.org.

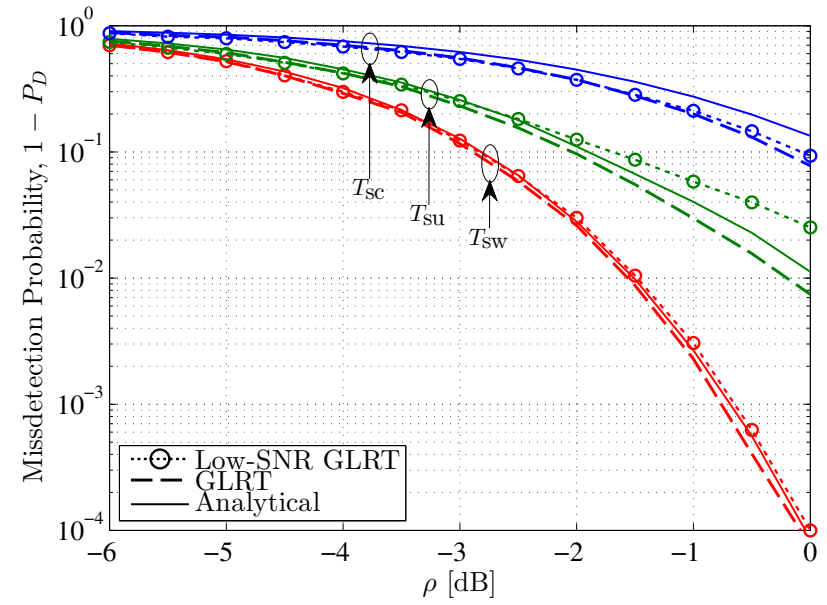

Fig. 4. 16-QAM signal with squared-root raised cosine pulse shaping (roll-off factor 1), for spatially i.i.d. noise (Model 1 ), $M=2, N=128, P_{\mathrm{FA}}=0.01$.

errors, a phenomenon also observed in previous detectors [6].

\section{B. All-SNR vs. low-SNR detectors}

It is worth noting that in Figs. 2 and 3, the performance of the all-SNR detector $T_{\mathrm{sw}}$ (resp. $T_{\mathrm{sc}}$ ), and that of the lowSNR detector $\Lambda_{\mathrm{sw}}^{\text {low }}$ (resp. $\Lambda_{\mathrm{sc}}^{\text {low }}$ ) coincide. This is due to the equivalence of (72) and (73) for binary PSDs. This is not the case though for the GLRT under Model 2, $T_{\mathrm{su}}$ and $\Lambda_{\mathrm{su}}^{\text {low }}$, or for other primary signal models. To see this, consider a 16-QAM primary signal with square-root raised cosine pulse shaping with roll-off factor 1 and the receiver bandwidth matching that of the signal. Hence, the sampling rate is 2 samples per QAM symbol. All other parameters are as in Fig. 2.

Fig. 4 shows the misdetection probability $1-P_{\mathrm{D}}$ versus $\rho$. While in this case none of the all-SNR detectors is exactly equivalent to its low-SNR approximation, the performance loss of $\Lambda_{\mathrm{sw}}^{\text {low }}$ and $\Lambda_{\mathrm{sc}}^{\text {low }}$ is negligible. Hence, these closed-form lowSNR detectors offer a good complexity-performance trade-off even when the primary signal PSD is non-binary. We can also appreciate their robustness to non-Gaussian primary signals.

Additionally, comparing Figs. 2 and 4, we note that for the 16-QAM scenario in Fig. 4, the performance of $T_{\mathrm{su}}$ and $T_{\mathrm{sw}}$ is still accurately predicted by the analytical expressions.

Comparing Figs. 2 and 4, in Fig. 4 we observe a degradation in the detection probability of the three detectors, moderate for $T_{\mathrm{sw}}$, and increasingly larger for $T_{\mathrm{su}}$ and $T_{\mathrm{sc}}$. This can be attributed to the effect of the spectral uniformity coefficient $\xi_{t}$ in the three detectors. For the signal in Fig. 2, we have $\xi_{t}=0.5$, while for the signal in Fig. $4, \xi_{t} \approx 0.67$. From the analysis in Section IV, the asymptotic performance of $T_{\mathrm{sw}}$, $T_{\mathrm{su}}$ and $T_{\mathrm{sc}}$ depends respectively on $\left(1-\frac{1}{M} \xi_{t}\right),\left(1-\xi_{t} \xi_{s}\right)$ and $\left(1-\xi_{t}\right)$, with $\frac{1}{M} \leq \xi_{s} \leq 1$. Hence, these detectors are increasingly sensitive to variations of the spectral uniformity.

\section{Detector performance vs. spatial selectivity $\xi_{s}$}

We now evaluate the performance of the detectors under Model 2 for particular per-antenna SNR profiles. We consider the GLR detector $T_{\mathrm{su}}$, and its particularizations: the selection

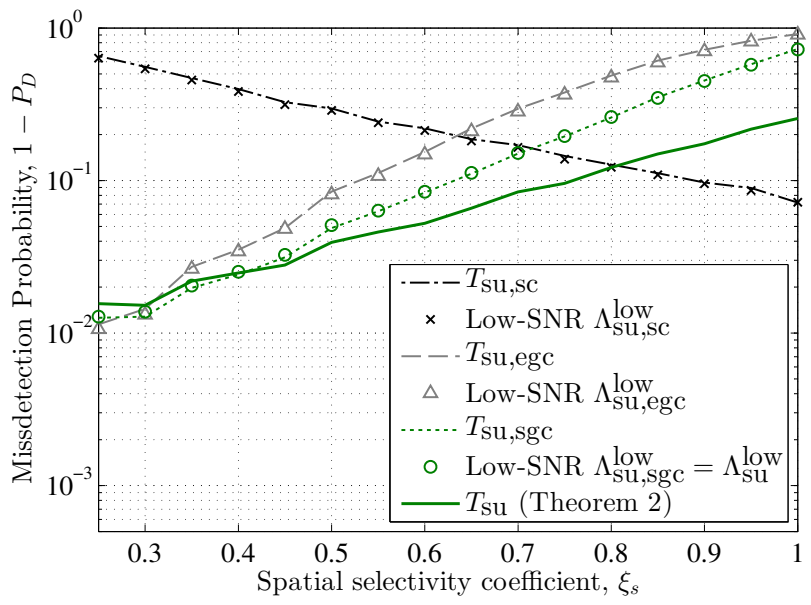

Fig. 5. Gaussian primary signal with binary PSD and spatially uncorrelated noise (Model 2), $\rho=1, M=4, N=64, P_{\text {FA }}=0.01$.

combining detector $T_{\mathrm{su} \text {-sc }}$, the equal gain combining detector $T_{\text {su-egc }}{ }^{3}$, and the similar gain combining detector $T_{\text {su-sgc }}$.

We consider a Gaussian primary signal with binary PSD occupying half of the bandwidth, and a diagonal $\boldsymbol{\Sigma}^{2}$. For each simulated point, we fix the per-antenna SNR profile such that $\rho_{1}$ and $\rho_{i}=\rho_{0}, i=2, \ldots, M$, are chosen to yield the desired spatial selectivity $\xi_{s}$. At each run, the diagonal coefficients of $\boldsymbol{\Sigma}^{2}$ are drawn from a $\chi_{1}^{2}$ distribution, and the phases of $\mathbf{h}$ are drawn, independently, from a uniform distribution $\mathcal{U}(0,2 \pi)$. We set $\rho=\sum_{i=1}^{M} \rho_{i}=1, M=4, N=64$, and $P_{\mathrm{FA}}=0.01$.

Fig. 5 shows the misdetection probability $1-P_{D}$ versus the spatial selectivity $\xi_{s}$. The corresponding low-SNR detectors are shown with markers. We can see that $T_{\text {su-sc }}$, derived assuming $\xi_{s}=1$, outperforms the other detectors when $\xi_{s}$ is indeed close to 1 . However, its performance degrades rapidly as $\xi_{s}$ decreases. Whereas detectors $T_{\text {su-sgc }}$ and $T_{\text {su-egc }}$ have similar performance as $\xi_{s} \rightarrow \frac{1}{M}, T_{\text {su-sgc }}$ outperforms $T_{\text {su-egc }}$ for $\xi_{s}>\frac{1}{M}$. For intermediate values of $\xi_{s}$ the GLRT $T_{\mathrm{su}}$ offers the best detection performance.

In Fig. 5 we can see that the performance of the all-SNR detectors $T_{\text {su-sc }}, T_{\text {su-egc }}$ and $T_{\text {su-sgc }}$ coincides with that of the corresponding low-SNR detectors, as the signal PSD is binary. Note however, that since $\Lambda_{\mathrm{su}}^{\mathrm{low}}=\Lambda_{\mathrm{su}-\mathrm{sgc}}^{\mathrm{low}}$ it does not coincide with that of $T_{\mathrm{su}}$, even for a binary PSD.

\section{Comparative evaluation for real communication signals}

The GLR tests in Section III exploit prior knowledge of the primary signal spectral shape. To show the advantage of this approach over other known detectors, consider a scenario based on the parameters of the GSM system [37]. We synthesize samples of a baseband Gaussian Minimum Shift Keying (GMSK) with a symbol rate of $270 \mathrm{~K}$ symbols/second, and a sampling rate of $400 \mathrm{kHz} .{ }^{4}$ Hence, we are considering

\footnotetext{
${ }^{3}$ The optimizer of $\boldsymbol{\psi}^{H} \mathbf{A} \boldsymbol{\psi}$ over $\boldsymbol{\psi}$ is approximated by using the phases of the eigenvector components associated with the largest eigenvalue of $\mathbf{A}$.

${ }^{4}$ The separation between GSM channels is $200 \mathrm{kHz}$, although in a given geographical area two adjacent channels cannot be active in order to avoid interference. Thus, the effective channel separation is $400 \mathrm{kHz}$, which is the same as the approximate bandwidth of the GSM signal. The PSD of the GMSK waveform is shown in the inset in Fig. 6.
} 
Copyright (c) 2016 IEEE. Personal use of this material is permitted. However, permission to use this material for any other purposes must be obtained from the IEEE by sending a request to pubs-permissions@ieee.org.

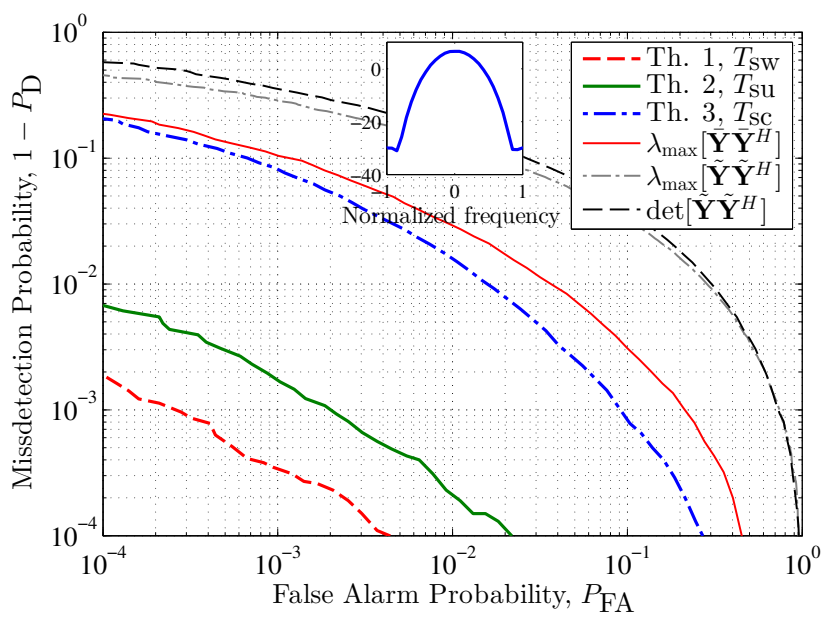

Fig. 6. GMSK primary signal and spatially i.i.d. noise (Model 1), $\rho=-3$ $\mathrm{dB}, M=4, N=256$. Inset: PSD of the GMSK signal.

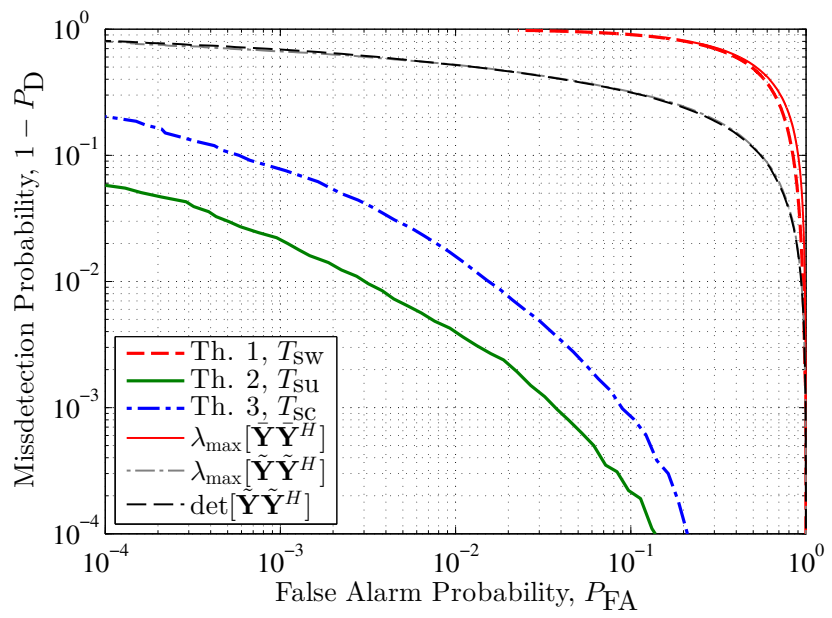

Fig. 7. GMSK primary signal and spatially uncorrelated noise (Model 2), $\rho=-3 \mathrm{~dB}, M=4, N=256$.

1.5 samples per symbol. At each run the channel coefficients are drawn from a Rayleigh distribution and are normalized to have a constant SNR $\rho=\mathbf{h}^{H} \boldsymbol{\Sigma}^{-2} \mathbf{h}$. We fix $M=4$ antennas, $N=256$ samples.

Figures 6 and 7 compare the receiver operating characteristic (ROC) curves of the tests $T_{\mathrm{sw}}, T_{\mathrm{su}}$ and $T_{\mathrm{sc}}$ with respect to existing detectors for a calibrated and an uncalibrated receiver, respectively. The following base-line tests are considered:

1) GLRT for temporally white Gaussian signals under Model 1, $\lambda_{\max }\left(\overline{\mathbf{Y}} \overline{\mathbf{Y}}^{H}\right)$ [4], [16]. This test corresponds to $T_{\mathrm{sw}}$ in (17)-(18) when $\mathbf{C}=\mathbf{I}_{N}$.

2) Low-SNR GLRT for temporally white Gaussian signals under Model 2, $\lambda_{\max }\left(\tilde{\mathbf{Y}} \tilde{\mathbf{Y}}^{H}\right)$ [6]. This test corresponds to $\Lambda_{\mathrm{su}}^{\text {low }}$ in (38) by setting $\mathbf{C}=\mathbf{I}_{N}$.

3) Rank-blind GLRT for temporally white Gaussian signals under Model $2, \operatorname{det}\left(\tilde{\mathbf{Y}} \tilde{\mathbf{Y}}^{H}\right)$ [3]. This test was shown to be the Locally Most Powerful Invariant Test at low-SNR for an unknown spatial rank of the primary signal [38].

Fig. 6 shows the ROC curves for spatially i.i.d. noise. $T_{\mathrm{sw}}$

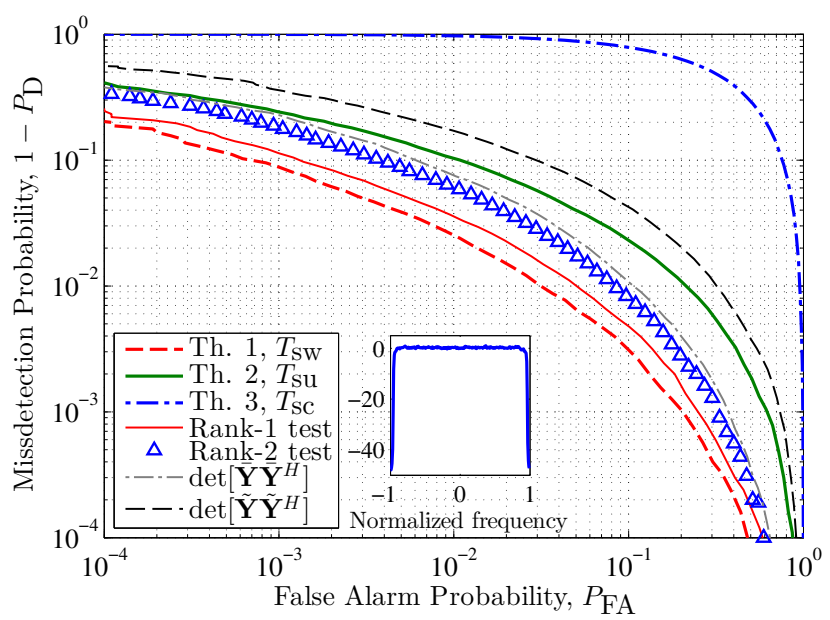

Fig. 8. Flat-fading channel model: DVB-T primary signal, spatially i.i.d. noise (Model 1), $\rho=-3 \mathrm{~dB}, M=4, N=256$. Inset: PSD of the DVB-T signal.

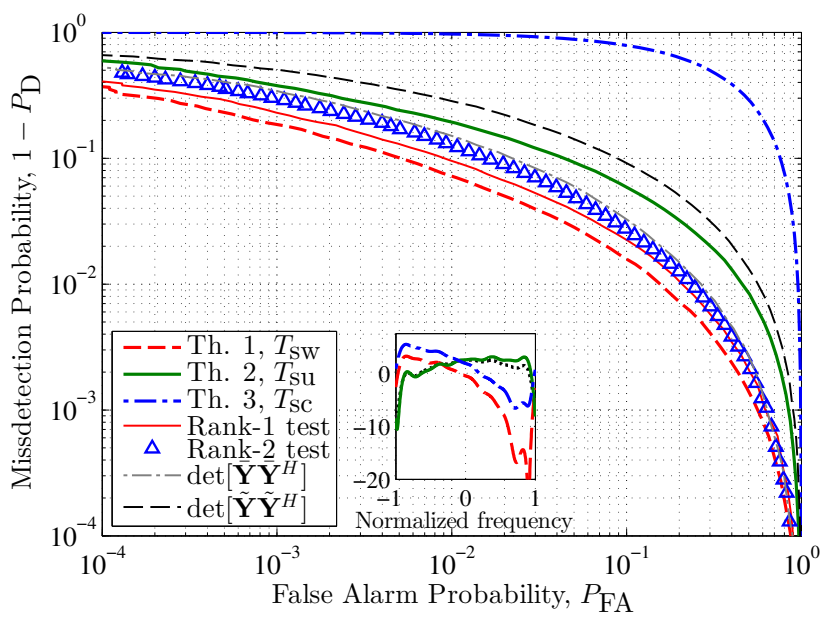

Fig. 9. WINNER II channel model: DVB-T primary signal, spatially i.i.d. noise (Model 1), $\rho=-3 \mathrm{~dB}, M=4, N=256$. Inset: One realization of the frequency selective channels to each of the antennas.

and $T_{\mathrm{su}}$ offer the best performance. Comparing the performance of the proposed detectors to that of the baseline detectors we can see the advantage of exploiting prior knowledge on the shape of the primary signal power spectral density. Fig. 7 shows the ROC curves for a receiver with different per-antenna noise power levels. In this setting, the detectors derived under noise Model $1\left(T_{\mathrm{sw}}\right.$ and $\left.\lambda_{\max }\left(\overline{\mathbf{Y}} \overline{\mathbf{Y}}^{H}\right)\right)$ degrade considerably, while $T_{\mathrm{su}}$ and $T_{\mathrm{sc}}$ offer the best performance.

\section{E. Effect of frequency-selective fading}

The model presented in Section II implicitly assumes frequency flat fading, since under frequency-selective fading the rank-1 assumption on the primary signal is no longer valid. Nevertheless, we show next that the proposed detectors are robust to frequency-selective fading in practical scenarios. We consider a $7.61 \mathrm{MHz}$-wide primary OFDM signal (DVB-T standard) with $8 \mathrm{MHz}$ channel separation (Fig. 8 inset), for which we study detection performance under flat-fading and 
Copyright (c) 2016 IEEE. Personal use of this material is permitted. However, permission to use this material for any other purposes must be obtained from the IEEE by sending a request to pubs-permissions@ieee.org.

under the WINNER Phase II channel model ${ }^{5}$ [39] (Fig. 9 inset shows one realization of the fading).

Additionally to the proposed detectors, we consider:

1) GLRT for rank- $P$ temporally white Gaussian signals under noise Model 1, [40, Eq. (11)], $P=1,2$.

2) Rank-blind GLRT for temporally white Gaussian signals under noise Model 1 [41], $\operatorname{det}\left(\overline{\mathbf{Y}} \overline{\mathbf{Y}}^{H}\right)$ (sphericity test).

3) Rank-blind GLRT for temporally white Gaussian signals under noise Model 2 [3], $\operatorname{det}\left(\tilde{\mathbf{Y}} \tilde{\mathbf{Y}}^{H}\right)$.

Figs. 8 and 9 show the ROC curves when the channel is frequency-flat and frequency-selective, respectively. We fix $M=4, N=256$, and we consider spatially i.i.d. noise. We can see that all detectors suffer a performance degradation under frequency selectivity. However, $T_{\mathrm{sw}}$ still offers the best detection performance, even under a deviation from the rank-1 model. The performance is in this case very close to that of the rank-1, rank-2 or rank-blind detectors derived for temporally white Gaussian signals. This is attributed to the fact that DVB$\mathrm{T}$ signals are frequency flat occupying most of the band, with spectral shape similar to that of a temporally white signal.

\section{CONCLUSIONS}

We have derived the GLRT for detecting Gaussian signals with known temporal correlation and unknown rankone spatial signature in additive, temporally white, spatially uncorrelated Gaussian noise. For spatially white (i.i.d.) noise, the GLRT boils down to a scalar optimization problem. For noises of disparate powers across antennas, we proposed to solve the problem by means of an alternating optimization algorithm. For low SNR, the GLRT admits a closed form under both models, and is shown to yield the exact all-SNR GLRT under particular conditions. When the primary signal is assumed temporally white, the proposed tests particularize to several detectors in the literature. However, exploiting prior knowledge of the signal temporal correlation significantly improves detection performance. We have provided an asymptotic analysis accurately quantifying this improvement. We have also shown that the derived low-SNR detectors are capable of operating under spatial rank larger than one for the primary signal.

\section{APPENDIX A}

\section{GLRT UNDER MODEL 1}

1) Proof of Theorem 1: Under Model 1 we can write $\Sigma^{2}=$ $\sigma^{2} \mathbf{I}_{M}$. Under $\mathcal{H}_{0}$, i.e. $\mathbf{h}=\mathbf{0}$, the ML estimation of the noise variance yields $\hat{\sigma}_{0}^{2}=\frac{1}{N M} \operatorname{tr}\left(\mathbf{Y} \mathbf{Y}^{H}\right)$. Substituting $\mathbf{h}=\mathbf{0}$ and $\boldsymbol{\Sigma}^{2}=\hat{\sigma}_{0}^{2} \mathbf{I}_{M}$ into (15), we get the compressed log-likelihood

$$
\ell_{0} \doteq-M N \log \frac{\pi e}{M N}-M N \log \operatorname{tr}\left(\mathbf{Y} \mathbf{Y}^{H}\right) .
$$

Under $\mathcal{H}_{1}$, the parameter space is given by $\Omega \doteq\left\{\mathbf{h}, \sigma^{2}\right\}$, with $\boldsymbol{\Sigma}^{2}=\sigma^{2} \mathbf{I}_{M}$. To derive the detector under $\mathcal{H}_{1}$ we operate

\footnotetext{
${ }^{5}$ Profile C1 (suburban), Non-line-of-sight (NLOS), central frequency 800 $\mathrm{MHz}$, channel bandwidth $8 \mathrm{MHz}$. Transmitter/receiver randomly distributed over a $10 \mathrm{~km}$-side square. $M=4$ linear receiver array with $10 \mathrm{~cm}$ separation.
}

instead in the transformed parameter space $\Omega^{\prime} \doteq\left\{\overline{\mathbf{h}}, \rho, \sigma^{2}\right\}$. The direct and inverse mappings are given respectively by,

$$
\begin{aligned}
& \Omega \rightarrow \Omega^{\prime} \quad: \quad \overline{\mathbf{h}}=\frac{\mathbf{h}}{\sqrt{\mathbf{h}^{H} \mathbf{h}}} \quad, \rho=\frac{\mathbf{h}^{H} \mathbf{h}}{\sigma^{2}}, \sigma^{2}=\sigma^{2} \\
& \Omega^{\prime} \rightarrow \Omega \quad: \quad \mathbf{h}=\overline{\mathbf{h}} \sqrt{\rho \sigma^{2}} \quad, \quad \sigma^{2}=\sigma^{2},
\end{aligned}
$$

where $\|\overline{\mathbf{h}}\|^{2}=\overline{\mathbf{h}}^{H} \overline{\mathbf{h}}=1$. The mapping between the two spaces is bijective and we may maximize the likelihood under $\mathcal{H}_{1}$ over either of the two spaces. Therefore, under $\mathcal{H}_{1}$ and in $\Omega^{\prime}$, we particularize (15) for $\boldsymbol{\Sigma}^{2}=\sigma^{2} \mathbf{I}_{M}$,

$$
\begin{array}{r}
\log f\left(\mathbf{Y} \mid \overline{\mathbf{h}}, \rho, \sigma^{2}\right)=-M N \log \left(\pi \sigma^{2}\right)-\log \operatorname{det}\left(\mathbf{I}_{N}+\rho \mathbf{C}\right) \\
-\frac{1}{\sigma^{2}} \operatorname{tr}\left(\mathbf{Y} \mathbf{Y}^{H}\right)+\frac{1}{\sigma^{2}} \rho \operatorname{tr}\left(\overline{\mathbf{h}} \overline{\mathbf{h}}^{H} \mathbf{Y} \mathbf{C}^{*}(\rho) \mathbf{Y}^{H}\right),(77)
\end{array}
$$

Setting the derivative of (77) vs. $\sigma^{2}$ equal to zero, we get $\hat{\sigma}_{1}^{2}=$ $\frac{1}{M_{2 N}} \operatorname{tr}\left(\mathbf{Y} \mathbf{Y}^{H}-\rho \overline{\mathbf{h}} \overline{\mathbf{h}}^{H} \mathbf{Y} \mathbf{C}^{*}(\rho) \mathbf{Y}^{H}\right)$ for the ML estimate of $\sigma^{2}$ under $\mathcal{H}_{1}$. Substituting $\hat{\sigma}_{1}^{2}$ into (77), we get

$$
\begin{array}{r}
\log f(\mathbf{Y} \mid \overline{\mathbf{h}}, \rho)=-M N \log \frac{\pi e}{M N}-\log \operatorname{det}\left(\mathbf{I}_{N}+\rho \mathbf{C}\right) \\
-M N \log \left(\operatorname{tr}\left(\mathbf{Y} \mathbf{Y}^{H}\right)-\rho \overline{\mathbf{h}}^{H} \mathbf{Y} \mathbf{C}^{*}(\rho) \mathbf{Y}^{H} \overline{\mathbf{h}}\right) .
\end{array}
$$

Its dependence on the unitary vector $\overline{\mathbf{h}}$ is restricted to the term $\overline{\mathbf{h}}^{H} \mathbf{Y} \mathbf{C}^{*}(\rho) \mathbf{Y}^{H} \overline{\mathbf{h}}$. Thus, the maximizing $\overline{\mathbf{h}}$ is the eigenvector associated with the largest eigenvalue of $\mathbf{Y} \mathbf{C}^{*}(\rho) \mathbf{Y}^{H}$. Substituting this value into (78), the log-likelihood under $\mathcal{H}_{1}$ can be written as a scalar optimization problem,

$$
\begin{aligned}
\ell_{1} \doteq \max _{\rho \geq 0}\left[-M N \log \frac{\pi e}{M N}-\log \operatorname{det}\left(\mathbf{I}_{N}+\rho \mathbf{C}\right)\right. \\
\left.-M N \log \left(\operatorname{tr}\left(\mathbf{Y} \mathbf{Y}^{H}\right)-\rho \lambda_{\max }\left(\mathbf{Y C}^{*}(\rho) \mathbf{Y}^{H}\right)\right)\right]
\end{aligned}
$$

Using that $T_{\mathrm{sw}}=\exp \left\{\ell_{1}-\ell_{0}\right\}$, Theorem 1 follows.

2) Asymptotic low-SNR GLRT: Consider the Taylor series expansion of $\log t_{\mathrm{sw}}(\rho)$ in Theorem 1 as $\rho \rightarrow 0^{+}: \log t_{\mathrm{sw}}(\rho)=$ $\sum_{i=1}^{\infty} a_{i} \rho^{i}$. In the low-SNR regime only the coefficient $a_{1}$ is relevant for the test (up to first order). In order to compute the coefficient $a_{1}$ in the series expansion we use that, as $\rho \rightarrow 0^{+}$,

$$
\begin{aligned}
\left(\mathbf{I}_{N}+\rho \mathbf{C}\right)^{-1} & =\mathbf{I}_{N}-\rho \mathbf{C}+o(\rho), \\
\operatorname{det}\left(\mathbf{I}_{N}+\rho \mathbf{C}\right) & =1+\rho \operatorname{tr} \mathbf{C}+o(\rho) .
\end{aligned}
$$

Substituting (81) in the definition of $t_{\mathrm{sw}}(\rho)$ in (18), applying the logarithm, we obtain

$$
\begin{aligned}
\log t_{\mathrm{sw}}(\rho) \\
=-M N \log \left(1-\rho \lambda_{\max }\left(\overline{\mathbf{Y}} \mathbf{C}^{*} \overline{\mathbf{Y}}^{H}-\rho \overline{\mathbf{Y}}\left(\mathbf{C}^{*}\right)^{2} \overline{\mathbf{Y}}^{H}+o(\rho)\right)\right) \\
\quad-\log \left(1+\rho \operatorname{tr} \mathbf{C}^{*}+o(\rho)\right) \\
=\rho\left(M N \lambda_{\max }\left(\overline{\mathbf{Y}} \mathbf{C}^{*} \overline{\mathbf{Y}}^{H}\right)-\operatorname{tr} \mathbf{C}^{*}\right)+o(\rho),
\end{aligned}
$$

where we used that $\log (1+a \rho)=a \rho+o(\rho)$. From (83), it follows that the first coefficient $a_{1}$ is given by

$$
a_{1}=M N \lambda_{\max }\left(\overline{\mathbf{Y}} \mathbf{C}^{*} \overline{\mathbf{Y}}^{H}\right)-\operatorname{tr} \mathbf{C}^{*} .
$$

For consistency with the maximization range $\rho \geq 0$ in (39), the low-SNR test in (20) is $\max \left\{0, a_{1}\right\}$. 
Copyright (c) 2016 IEEE. Personal use of this material is permitted. However, permission to use this material for any other purposes must be obtained from the IEEE by sending a request to pubs-permissions@ieee.org.

3) Performance analysis: The asymptotic performance of the test $T_{\mathrm{sw}}$ is characterized by the non-centrality parameter $\alpha_{\mathrm{sw}}=\mathbb{E}\left[2 \log T_{\mathrm{sw}} \mid \mathcal{H}_{1}\right]$. To compute this expectation, we recover the property in [12, Eq. 76] concerning convergence in variance of sample spatial correlations when $N \rightarrow \infty$,

$$
\frac{1}{N} \mathbf{Y A Y}^{H} \rightarrow \frac{1}{N} \operatorname{tr}(\mathbf{A}) \cdot \boldsymbol{\Sigma}^{2}+\frac{1}{N} \operatorname{tr}\left(\mathbf{C}^{*} \mathbf{A}\right) \cdot \mathbf{h h}^{H} .
$$

with $\mathbf{A}$ a temporal correlation matrix. From (85) one has

$$
\begin{aligned}
\mathbf{Y} \mathbf{C}^{*}(\rho) \mathbf{Y}^{H} & \rightarrow \operatorname{tr}\left(\mathbf{C}^{*}(\rho)\right) \boldsymbol{\Sigma}^{2}+\operatorname{tr}\left(\mathbf{C}^{*} \mathbf{C}^{*}(\rho)\right) \mathbf{h} \mathbf{h}^{H}, \\
\operatorname{tr}\left(\mathbf{Y} \mathbf{Y}^{H}\right) & \rightarrow N M \sigma^{2}+\|\mathbf{h}\|^{2} \operatorname{tr} \mathbf{C}^{*},
\end{aligned}
$$

as $N \rightarrow \infty$, where we used that $\operatorname{tr}\left(\boldsymbol{\Sigma}^{2}\right)=\operatorname{tr}\left(\sigma^{2} \mathbf{I}_{M}\right)=M \sigma^{2}$. From (86) and (87) we obtain

$$
\frac{\lambda_{\max }\left(\mathbf{Y} \mathbf{C}^{*}(\rho) \mathbf{Y}^{H}\right)}{\operatorname{tr}\left(\mathbf{Y} \mathbf{Y}^{H}\right)} \rightarrow \frac{\sigma^{2} \operatorname{tr}\left(\mathbf{C}^{*}(\rho)\right)+\|\mathbf{h}\|^{2} \operatorname{tr}\left(\mathbf{C}^{*} \mathbf{C}^{*}(\rho)\right)}{N M \sigma^{2}+\|\mathbf{h}\|^{2} \operatorname{tr} \mathbf{C}^{*}} .
$$

Now, with $\lambda_{k}$ the eigenvalues of $\mathbf{C}$, it follows that

$$
\begin{aligned}
& \operatorname{tr}\left(\mathbf{C}^{*}(\rho)\right)+\rho \operatorname{tr}\left(\mathbf{C}^{*} \mathbf{C}^{*}(\rho)\right) \\
& =\sum_{k=1}^{N} \frac{\lambda_{k}}{1+\rho \lambda_{k}}+\rho \sum_{k=1}^{N} \frac{\lambda_{k}^{2}}{1+\rho \lambda_{k}}=\sum_{k=1}^{N} \lambda_{k}=\operatorname{tr} \mathbf{C}^{*} .
\end{aligned}
$$

Given the consistency of ML estimators, the estimate of $\rho$ converges to its true value as $N \rightarrow \infty$. Then, for $\rho \rightarrow\|\mathbf{h}\|^{2} / \sigma^{2}$, from (88) and (89) we obtain,

$$
\frac{\lambda_{\max }\left(\mathbf{Y} \mathbf{C}^{*}(\rho) \mathbf{Y}^{H}\right)}{\operatorname{tr}\left(\mathbf{Y} \mathbf{Y}^{H}\right)} \rightarrow \frac{\operatorname{tr} \mathbf{C}^{*}}{N M+\rho \operatorname{tr} \mathbf{C}^{*}} .
$$

Substituting (90) into (17)-(18), yields

$$
\alpha_{\mathrm{sw}} \rightarrow 2 N \log \frac{\left(1+\frac{\rho}{N M} \operatorname{tr} \mathbf{C}^{*}\right)^{M}}{\operatorname{det}\left(\mathbf{I}_{N}+\rho \mathbf{C}\right)^{\frac{1}{N}}} .
$$

In our model, $\operatorname{tr} \mathbf{C}^{*}=\operatorname{tr} \mathbf{C}=N$. Then, (44) follows.

\section{APPENDIX B}

\section{GLRT UNDER MODEL 2}

1) Proof of Proposition 1: The terms in (31) depending on $\mathbf{T}$ are given by

$$
J(\mathbf{T} \mid \overline{\boldsymbol{v}}, \rho) \doteq \log \operatorname{det}\left(\mathbf{T}^{2}\right)-\operatorname{tr}\left(\mathbf{T}^{2}\right)+\frac{\beta}{\overline{\boldsymbol{v}}^{H} \mathbf{T}^{-2} \overline{\boldsymbol{v}}},
$$

with $\beta=\beta(\overline{\boldsymbol{v}}, \rho)$. Using that $\mathbf{T}$ is a diagonal matrix, we express $J(\mathbf{T})$ in scalar form as

$$
J(\mathbf{T} \mid \overline{\boldsymbol{v}}, \rho)=\sum_{i=1}^{M}\left(\log t_{i}^{2}-t_{i}^{2}\right)+\beta\left(\sum_{i=1}^{M}\left|\bar{v}_{i}\right|^{2} t_{i}^{-2}\right)^{-1} .
$$

Taking the derivative of $J(\mathbf{T} \mid \overline{\boldsymbol{v}}, \rho)$ with respect to $t_{i}^{2}$ yields

$$
\begin{aligned}
\frac{\partial}{\partial t_{i}^{2}} J(\mathbf{T} \mid \overline{\boldsymbol{v}}, \rho) & =t_{i}^{-2}-1+\frac{1}{4} \phi^{2}\left|\bar{v}_{i}\right|^{2} t_{i}^{-4}, \\
\phi & \doteq 2 \sqrt{\beta}\left(\sum_{i=1}^{M}\left|\bar{v}_{i}\right|^{2} t_{i}^{-2}\right)^{-1} .
\end{aligned}
$$

Equating (94) to zero for each $i=1, \ldots, M$ we obtain the following nonlinear system of equations, as well as its solution

$$
\begin{aligned}
& t_{i}^{4}-t_{i}^{2}-\frac{1}{4} \phi^{2}\left|\bar{v}_{i}\right|^{2}=0, \quad i=1, \ldots, M . \\
& \Rightarrow t_{i}^{2}=\frac{1}{2}\left(1+\sqrt{1+\phi^{2}\left|\bar{v}_{i}\right|^{2}}\right),
\end{aligned}
$$

The solution of (96) in (97) can be shown to be a maximum of $J(\mathbf{T})$. In particular, we have discarded the negative solution, as $t_{i}^{2}$ must be positive. Note that (97) is an implicit equation since $\phi$ depends on $t_{i}^{2}, i=1, \ldots, M$. The value of $\phi$ can be obtained as follows. Substituting (97) in (95) we obtain

$$
\begin{aligned}
\phi & =2 \sqrt{\beta}\left(\sum_{i=1}^{M} 2\left|\bar{v}_{i}\right|^{2}\left(1+\sqrt{1+\phi^{2}\left|\bar{v}_{i}\right|^{2}}\right)^{-1}\right)^{-1} \\
& =\sqrt{\beta} \phi^{2}\left(\sum_{i=1}^{M}\left(\sqrt{1+\phi^{2}\left|\bar{v}_{i}\right|^{2}}-1\right)\right)^{-1}
\end{aligned}
$$

where, multiplying and dividing by $\sqrt{1+\phi^{2}\left|\bar{v}_{i}\right|^{2}}-1$ each term in the sum in (98), we obtain (99). Thus, from (99), $\phi$ is defined as an implicit function in terms of $\beta$ and $\overline{\boldsymbol{v}}$ as

$$
\sqrt{\beta}=\phi^{-1} \sum_{i=1}^{M}\left(\sqrt{1+\phi^{2}\left|\bar{v}_{i}\right|^{2}}-1\right) .
$$

The first part of Proposition 1 follows from (97) and (100).

To prove the second part, we multiply (96) by $t_{i}^{-2}$ and sum the set of resulting equations for $i=1, \ldots, M$. We obtain

$$
\sum_{i=1}^{M}\left(t_{i}^{2}-1\right)=\frac{\phi^{2}}{4} \sum_{i=1}^{M} \frac{\left|\bar{v}_{i}\right|^{2}}{t_{i}^{2}}=\beta\left(\sum_{i=1}^{M} \frac{\left|\bar{v}_{i}\right|^{2}}{t_{i}^{2}}\right)^{-1},
$$

where we used that $\phi^{2}=4 \beta\left(\sum_{i=1}^{M}\left|\bar{v}_{i}\right|^{2} t_{i}^{-2}\right)^{-2}$. Writing the sum in (101) in matrix form, (35) follows.

2) Asymptotic low-SNR GLRT: Let us define

$$
\begin{aligned}
\lambda_{\text {su }}(\overline{\boldsymbol{v}}, \rho) & \doteq \log t_{\text {su }}(\overline{\boldsymbol{v}}, \rho) \\
& =N \log \operatorname{det}\left(\mathbf{T}^{2}(\overline{\boldsymbol{v}}, \rho)\right)-\log \operatorname{det}\left(\mathbf{I}_{N}+\rho \mathbf{C}\right) .
\end{aligned}
$$

The low-SNR GLR detector follows from considering the first term in the low-SNR expansion of $\lambda_{\mathrm{su}}(\overline{\boldsymbol{v}}, \rho)$,

$$
\Lambda_{\text {su }}^{\text {low }} \doteq \max \left\{0, \max _{\overline{\boldsymbol{v}}} \lim _{\rho \rightarrow 0^{+}} \frac{1}{\rho} \lambda_{\mathrm{su}}(\overline{\boldsymbol{v}}, \rho)\right\} .
$$

To compute $\Lambda_{\mathrm{su}}^{\text {low }}$, we first derive an alternative form for $\lambda_{\text {su }}(\overline{\boldsymbol{v}}, \rho)$. Integrating $\tau(q)$ in (34) in Proposition 1 yields

$$
\begin{aligned}
& \int \tau(q) \mathrm{d} q=\sum_{i=1}^{M} \int \frac{-1+\sqrt{1+\left|\bar{v}_{i}\right|^{2} q^{2}}}{q} \mathrm{~d} q \\
& =c+\sum_{i=1}^{M}\left(\sqrt{1+\left|\bar{v}_{i}\right|^{2} q^{2}}-\log \left(1+\sqrt{1+\left|\bar{v}_{i}\right|^{2} q^{2}}\right)\right),
\end{aligned}
$$

for an arbitrary constant $c$, where the last step follows from [42, Eq. 17.9.12]. Also from (34) it follows that

$$
q \cdot \tau(q)=\sum_{i=1}^{M}\left(\sqrt{1+q^{2}\left|\bar{v}_{i}\right|^{2}}-1\right) .
$$

Consider now an arbitrary one-to-one function $\tau=\tau(q)$, with $q=q(\tau)$ its inverse. It is easy to check that the area of the rectangle $[0, q] \times[0, \tau(q)]$ can be decomposed as

$$
q \cdot \tau(q)=\int_{0}^{q} \tau\left(q^{\prime}\right) \mathrm{d} q^{\prime}+\int_{0}^{\tau(q)} q\left(\tau^{\prime}\right) \mathrm{d} \tau^{\prime} .
$$

Then, by combining (104), (105) and (106) we obtain

$$
\begin{aligned}
\int_{0}^{\tau} q\left(\tau^{\prime}\right) \mathrm{d} \tau^{\prime} & =\sum_{i=1}^{M} \log \left(1+\sqrt{1+p_{i} q^{2}}\right) \\
& =\log \operatorname{det}\left(\mathbf{T}^{2}(\overline{\boldsymbol{v}}, \rho)\right),
\end{aligned}
$$

where (108) follows from Proposition 1 for $q=q(\sqrt{\beta(\overline{\boldsymbol{v}}, \rho)})$. Substituting (108) in (102), we get the integral form

$$
\lambda_{\mathrm{su}}(\overline{\boldsymbol{v}}, \rho)=N \int_{0}^{\sqrt{\beta(\overline{\boldsymbol{v}}, \rho)}} q\left(\tau^{\prime}\right) \mathrm{d} \tau^{\prime}-\log \operatorname{det}\left(\mathbf{I}_{N}+\rho \mathbf{C}\right)
$$


Copyright (c) 2016 IEEE. Personal use of this material is permitted. However, permission to use this material for any other purposes must be obtained from the IEEE by sending a request to pubs-permissions@ieee.org.

for $\lambda_{\mathrm{su}}(\overline{\boldsymbol{v}}, \rho)$. For small $\tau$, from (34) we obtain $q(\tau)=2 \tau+$ $o(\tau)$, which integrates to $\tau^{2}+o\left(\tau^{2}\right)$. Hence (109) becomes

$$
\lambda_{\text {su }}(\overline{\boldsymbol{v}}, \rho)=N \beta(\overline{\boldsymbol{v}}, \rho)-\log \operatorname{det}\left(\mathbf{I}_{N}+\rho \mathbf{C}\right)+o(\beta(\overline{\boldsymbol{v}}, \rho)) .
$$

At low SNR $\mathbf{C}(\rho)=\underset{\tilde{\mathbf{C}}}{\mathbf{C}}+o(\rho)$. Then, it follows that $\beta(\overline{\boldsymbol{v}}, \rho)=\frac{1}{N} \overline{\boldsymbol{v}}^{H} \tilde{\mathbf{Y}}^{H} \rho \mathbf{C}^{*} \tilde{\mathbf{Y}} \overline{\boldsymbol{v}}+o(\rho)$. Substituting the lowSNR expansions for $\beta(\overline{\boldsymbol{v}}, \rho)$ and using that $\log \operatorname{det}(\mathbf{I}+\rho \mathbf{C})=$ $\rho \operatorname{tr}(\mathbf{C})+o(\rho)$ in (110), via (103) we obtain

$$
\Lambda_{\mathrm{su}}^{\text {low }}=\max \left\{0, \lambda_{\max }\left(\tilde{\mathbf{Y}} \mathbf{C}^{*} \tilde{\mathbf{Y}}^{H}\right)-\operatorname{tr} \mathbf{C}\right\} .
$$

3) Performance analysis: Under Model 2, the GLRT performance depends on the non-centrality parameter

$$
\alpha_{\mathrm{su}}=\mathbb{E}\left[2 \log T_{\mathrm{su}} \mid \mathcal{H}_{1}\right] .
$$

Using the property (85) it follows that, for $N \rightarrow \infty$,

$$
\mathbf{D}^{2}=\operatorname{diag}\left(\frac{1}{N} \mathbf{Y} \mathbf{Y}^{H}\right) \rightarrow \operatorname{diag}\left(\boldsymbol{\Sigma}^{2}+\frac{\operatorname{tr} \mathbf{C}^{*}}{N} \cdot \mathbf{h} \mathbf{h}^{H}\right)
$$

Thus, from the definition of $\mathbf{T}$ in (25), and using the asymptotic consistency of the ML estimator, we obtain that $t_{i}^{2} \rightarrow 1+\frac{\operatorname{tr} \mathbf{C}^{*}}{N} \rho_{i}$, where $\rho_{i}=\frac{\left|h_{i}\right|^{2}}{\sigma_{i}^{2}}$. Substituting this result in the detector $T_{\mathrm{su}}$ in (36)-(37), from the definition (112) we get

$$
\alpha_{\mathrm{su}} \rightarrow 2 N \log \frac{\prod_{i=1}^{M}\left(1+\frac{\operatorname{tr} \mathbf{C}^{*}}{N} \rho_{i}\right)}{\operatorname{det}\left(\mathbf{I}_{N}+\rho \mathbf{C}\right)^{\frac{1}{N}}} .
$$

Eq. (45) follows using that $\operatorname{tr} \mathbf{C}^{*}=\operatorname{tr} \mathbf{C}=N$.

\section{APPENDIX C}

\section{DERIVATION OF (72) FOR BINARY POWER SPECTRUM}

For ease of exposition let us define $\varrho \doteq(1+\rho \mu)^{-1}$. The optimization in (71) in terms of $\rho$ can be rewritten as

$$
\begin{aligned}
T_{\mathrm{bs}} & =\max _{0<\varrho \leq 1} \exp \{g(\varrho)\}, \\
g(\varrho) & \doteq-L \log (1-(1-\varrho) b)+r \log \varrho,
\end{aligned}
$$

in terms of $\varrho$. To obtain the maximizing $\varrho$ in (115) we first differentiate $g(\varrho)$, where: $g^{\prime}(\varrho)=\frac{-L b}{1-(1-\varrho) b}+\frac{r}{\varrho}$. Equating $g^{\prime}(\varrho)$ to 0 we obtain the optimality condition

$$
\varrho=\frac{1}{L-r} \cdot r\left(b^{-1}-1\right) .
$$

As $0<\varrho \leq 1$, this condition holds provided that $b \geq r / L$. On the other hand, for $b<r / L$, we have that

$$
g^{\prime}(\varrho)=\frac{L}{\varrho}\left(\frac{r}{L}-\left(1-\frac{1-b}{1-b+\varrho b}\right)\right) \geq \frac{L}{\varrho}\left(\frac{r}{L}-b\right) \geq 0,
$$

where in (118) we used that $\varrho \leq 1$ and that $b \leq 1$ for the detectors in Table II. Therefore, under the stated conditions, $g(\varrho)$ is nondecreasing in $0<\varrho \leq 1$ and its maximum is attained at $\varrho=1$. Thus, from (117) (when $b \geq r / L$ ) and from (118) (when $0 \leq b<r / L$ ), the $\varrho$ maximizing (115) is given by $\varrho_{\star}=\max \left\{1, \frac{1}{L-r} \cdot r\left(b^{-1}-1\right)\right\}$. Substituting $\varrho=\varrho_{\star}$ in (115), we get (72). The optimal value of $\rho$ in (71) follows from $\varrho=(1+\rho \mu)^{-1}$, as $\rho_{\star}=\frac{1-\varrho_{\star}}{\mu \varrho_{\star}}$.

\section{REFERENCES}

[1] E. Axell, G. Leus, E. Larsson, and H. Poor, "Spectrum sensing for cognitive radio: State-of-the-art and recent advances," IEEE Signal Process. Mag., vol. 29, no. 3, pp. 101 - 116, May 2012.

[2] D. Hack, L. Partton, and B. Himed, "Multichannel detection of an unknown rank-one signal with uncalibrated receivers," in IEEE Int. Conf. Acoustics, Speech, Signal Process., Florence, Italy, May 2014.

[3] A. Leshem and A.-J. van der Veen, "Multichannel detection of Gaussian signals with uncalibrated receivers," IEEE Signal Process. Lett., vol. 8, no. 4, pp. 120-122, Apr. 2001.

[4] A. Taherpour, M. Nasiri-Kenari, and S. Gazor, "Multiple antenna spectrum sensing in cognitive radios," IEEE Trans. Wireless Commun., vol. 9, no. 2, pp. $814-823$, Feb. 2010.

[5] S. Sedighi, A. Taherpour, and J. Sala, "Spectrum sensing using correlated receiving multiple antennas in cognitive radios," IEEE Trans. Wireless Commun., vol. 12, no. 11, pp. 5754 - 5766, Nov. 2013.

[6] R. López-Valcarce, G. Vazquez-Vilar, and J. Sala, "Multiantenna spectrum sensing for cognitive radio: overcoming noise uncertainty," in Int. Workshop Cognitive Inf. Process. (CIP), Elba, Italy, Jun. 2010, pp. 310 -315 .

[7] A. I. Pérez-Neira, M. A. Lagunas, M. A. Rojas, and P. Stoica, "Correlation matching approach for spectrum sensing in open spectrum communications," IEEE Trans. Signal Process., vol. 57, no. 12, pp. 4823 - 4836, Dec. 2009.

[8] E. Lagunas and M. Nájar, "Sparse correlation matching-based spectrum sensing for open spectrum communications," EURASIP J. Advances Signal Process., pp. 1 - 14, Feb. 2012.

[9] J. Font-Segura, G. Vàzquez, and J. Riba, "Compressed correlationmatching for spectrum sensing in sparse wideband regimes," in IEEE Int. Conf. Commun., Jun. 2011, pp. $1-5$.

[10] E. Lagunas and M. Nájar, "Spectral feature detection with sub-Nyquist sampling for wideband spectrum sensing," IEEE Trans. Wireless Commun., vol. 14, no. 7, pp. 3978 - 3990, Jul. 2015.

[11] G. Vazquez-Vilar, R. López-Valcarce, and J. Sala, "Multiantenna spectrum sensing exploiting spectral a priori information," IEEE Trans. Wireless Commun., vol. 10, no. 12, pp. 4345-4355, Dec. 2011.

[12] J. Sala-Álvarez, G. Vazquez-Vilar, and R. López-Valcarce, "Multiantenna GLR detection of rank-one signals with known power spectrum in white noise with unknown spatial correlation," IEEE Trans. Signal Process., vol. 60, no. 6, pp. 3065 -3078, Jun. 2012.

[13] I. Akyildiz, W.-Y. Lee, M. Vuran, and S. Mohanty, "A survey on spectrum management in cognitive radio networks," IEEE Commun. Mag., vol. 46, no. 4, pp. 40-48, Apr. 2008.

[14] J. M. Peha, "Sharing spectrum through spectrum policy reform and cognitive radio," Proc. IEEE, vol. 97, no. 4, pp. 708-719, Apr. 2009.

[15] J. Lundén, V. Koivumen, and H. Poor, "Spectrum exploration and exploitation for cognitive radio: Recent advances," IEEE Signal Process. Mag., vol. 32, no. 3, pp. 123 - 140, May 2015.

[16] O. Besson, S. Kraut, and L. Scharf, "Detection of an unknown rank-one component in white noise," IEEE Trans. Signal Process., vol. 54, no. 7, pp. 2835 - 2839, Jul. 2006.

[17] D. Romero and R. López-Valcarce, "Spectrum sensing for wireless microphone signals using multiple antennas," IEEE Trans. Veh. Technol., vol. 63, no. 9, pp. 4395-4407, Nov 2014.

[18] P. Wang, J. Fang, N. Han, and H. Li, "Multiantenna-assisted spectrum sensing for cognitive radio," IEEE Trans. Veh. Technol., vol. 59, no. 4 , pp. 1791-1800, May 2010.

[19] D. Ramírez, J. Vía, and I. Santamaría, "Multiantenna spectrum sensing: The case of wideband rank-one primary signals," in IEEE Sensor Array Multichannel Signal Process. Workshop, Israel, Oct. 2010, pp. 9 - 12.

[20] R. Zhang, T. Lim, Y.-C. Liang, and Y. Zeng, "Multi-antenna based spectrum sensing for cognitive radios: A GLRT approach," IEEE Trans. Commun., vol. 58, no. 1, pp. 84-88, Jan. 2010.

[21] H. D. Griffiths and C. J. Baker, "Passive coherent location radar systems. part 1: Performance prediction," IEE Proc. - Radar, Sonar Navig., vol. 152, no. 3, pp. 153 - 159, Jun. 2005.

[22] C. J. Baker, H. D. Griffiths, and I. Papoutsis, "Passive coherent location radar systems. part 2: Waveform properties," IEE Proc. - Radar, Sonar Navig., vol. 152, no. 3, pp. 160 - 168, Jun. 2005.

[23] D. E. Hack, L. K. Patton, B. Himed, and M. A. Saville, "Detection in passive MIMO radar networks," IEEE Trans. Signal Process., vol. 62, no. 11, pp. 2999 - 3012, Jun. 2014.

[24] G. Cui, J. Liu, H. Li, and B. Himed, "Signal detection with noisy reference for passive sensing," Signal Process., vol. 108, pp. 389 - 399, Mar. 2015. 
Copyright (c) 2016 IEEE. Personal use of this material is permitted. However, permission to use this material for any other purposes must be obtained

[25] J. Liu, H. Li, and B. Himed, "Two target detection algorithms for passive multistatic radar," IEEE Trans. Signal Process., vol. 62, no. 22, pp. 5930 - 5939, Nov. 2014

[26] T. Yücek and H. Arslan, "A survey of spectrum sensing algorithms for cognitive radio applications," IEEE Communications Surveys and Tutorials, vol. 11, no. 1, pp. 116-130, First quarter 2009.

[27] S. M. Kay, Fundamentals of Statistical Signal Processing, Volume II: Detection Theory. Englewood Cliffs, N.J.: Prentice-Hall, 1998.

[28] H. van Trees, Optimum Array Processing (Detection, Estimation and Modulation Theory, Part IV). John Wiley and Sons Inc., 2002.

[29] L. Huang, Y. Xiao, and Q. Zhang, "Robust spectrum sensing for noncircular signal in multiantenna cognitive receivers," IEEE Trans. Signal Process., vol. 63, no. 2, pp. 498 - 511, Jan. 2015.

[30] J. Villares, Sample Covariance Based Parameter Estimation for Digital Communications. $\mathrm{PhD}$. Dissertation, Universitat Politècnica de Catalunya, 2005.

[31] J. Villares and G. Vàzquez, "The Gaussian assumption in second-order estimation problems in digital communications," IEEE Trans. Signal Process., vol. 55, no. 10, pp. 4994 - 5002, Oct. 2007.

[32] S. M. Kay, Fundamentals of Statistical Signal Processing: Detection Theory. Englewood Cliffs, NJ: Prentice-Hall, 1998.

[33] R. M. Gray, Toeplitz and Circulant Matrices: A Review. New York: Hanover/Now, 2006

[34] R. Tandra and A. Sahai, "SNR walls for signal detection," IEEE J. Sel. Top. Signal Process., vol. 2, no. 1, pp. 4-17, Feb 2008

[35] D. S. Bernstein, Matrix Mathematics: Theory, Facts and Formulas (2nd ed.). Princeton University Press, 2009.

[36] Y. Zeng, Y.-C. Liang, and R. Zhang, "Blindly combined energy detection for spectrum sensing in cognitive radio," IEEE Signal Processing Lett., vol. 15, pp. 649-652, 2008

[37] R. Steele, Mobile Radio Communications. Pentech Press, 1992.

[38] D.Ramírez, J.Vía, and I.Santamaría, "The locally most powerful test for multiantenna spectrum sensing with uncalibrated receivers," in IEEE Int. Conf. Acoustics, Speech, Signal Process., Kyoto, Japan, Mar. 2012, pp. $3437-3440$.

[39] L. Hentilä, P. Kyösti, M. Käske, M. Narandzic, and M. Alatossava, "MATLAB implementation of the WINNER Phase II Channel Model ver 1.1," Online: https://www.ist-winner.org/phase_2_model.html, Dec. 2007.

[40] D. Ramírez, G. Vazquez-Vilar, R. López-Valcarce, J. Vía, and I. Santamaría, "Detection of rank-P signals in cognitive radio networks with uncalibrated multiple antennas," IEEE Trans. Signal Process., vol. 59, no. 8, pp. 3764-3774, Aug. 2011.

[41] J. Mauchly, "Significance test for sphericity of a normal n-variate distribution," Ann. Math. Statist., vol. 11, no. 2, pp. $204-$ 209, 1940.

[42] S. Lipschutz, M. Spiegel, and J. Liu, Mathematical Handbook of Formulas and Tables (3rd ed.). McGraw-Hill, 2009.

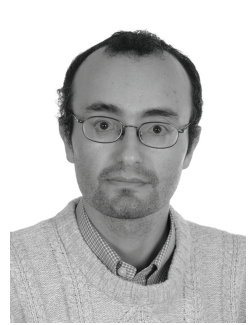

Josep Sala-Alvarez (S'86 - SM'11) born in Barcelona, Spain, in 1967. He received the M.Sc. and Ph.D. degrees in telecommunications engineering from the Technical University of Catalonia (UPC), Barcelona, Spain, in 1991 and 1995, respectively. During 1992, he worked at the European Space Operations Centre (ESOC) of the European Space Agency (ESA), Darmstadt, Germany, in the area of software engineering for telemetry processing. From 1993 to late 1994, he held a grant from the Generalitat de Catalunya in support of the Ph.D. degree at the Department of Signal Theory and Communications, UPC. In 1994, he joined this department as Assistant Professor and was promoted to Associate Professor in 1997. He has participated in space-related communication projects for ESA and in wireless communications projects at the national/European level with industry and institutions. His current research interests are in the field of signal processing, communications, and information theory. Dr. Sala is recipient of the IEEE Signal Processing Society Best (Senior) PaperAward 2003, the International Symposium on Turbo-Codes and Applications (ISTCâÁZ03) Best Poster Paper Award, and the Best Ph.D. Thesis in Telecommunications National Award (Spain, 1995). Dr. Sala is an IEEE Senior Member.

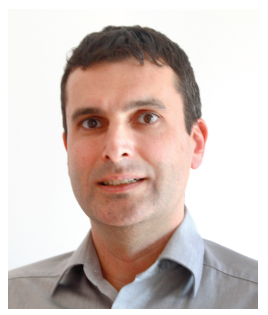

Gonzalo Vazquez-Vilar (S'08, M'12) received the Telecommunication Engineering degree from the University of Vigo, Spain, in 2004, the Master of Science degree from Stanford University, U.S., in 2008 and the Ph.D. in Communication Systems from the University of Vigo, Spain, in 2011. In 2011-2014 he was a post-doctoral fellow in the Department of Information and Communication Technologies, Universitat Pompeu Fabra, Spain and since 2014 he has been a Visiting Professor in the Department of Signal Theory and Communications, Universidad

Carlos III de Madrid, Spain

He has held appointments as visiting researcher at Stanford University, U.S., University of Cambridge, U.K., and Princeton University, U.S. His research interests lie in the field of Shannon theory, with emphasis on finite-length information theory and communications.

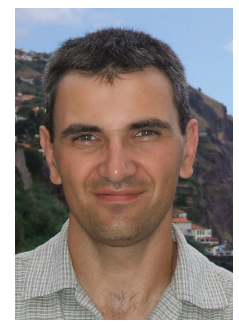

Roberto López-Valcarce (S'95-M'01) received the Telecommunication Engineering degree from the University of Vigo, Vigo, Spain, in 1995, and the M.S. and Ph.D. degrees in electrical engineering from the University of Iowa, Iowa City, in 1998 and 2000, respectively. During 1995 he was a Project Engineer with Intelsis. He was a Ramón y Cajal Postdoctoral Fellow of the Spanish Ministry of Science and Technology from 2001 to 2006. During that period, he was with the Signal Theory and Communications Department, University of Vigo, where he currently is an Associate Professor. His main research interests include adaptive signal processing, digital communications, and sensor networks, having coauthored over 50 papers in leading international journals. He holds several patents in collaboration with industry.

Dr. López-Valcarce was a recipient of a 2005 Best Paper Award of the IEEE Signal Processing Society. He served as an Associate Editor of the IEEE TRANSACTIONS ON SIGNAL PROCESSING from 2008 to 2011, and as a member of the IEEE Signal Processing for Communications and Networking Technical Committee from 2011 to 2013.

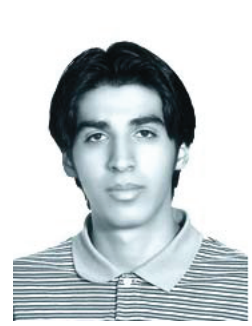

Saeid Sedighi was born in Tehran, Iran, in 1987. He received the B.Sc. degree in Electrical Engineering from the Islamic Azad University, Tehran, Iran, in 2010 and the M.Sc. degree in Electrical Engineering, Telecommunications, from Imam Khomeini International University (IKIU), Qazvin, Iran, in 2012. Since October 2012, he has been serving as a research assistant in the Signal Processing and Information Theory (SPIT) lab at the Electrical Engineering Department, IKIU. His research interests are cognitive radio, statistical signal processing, detection and estimation theories.

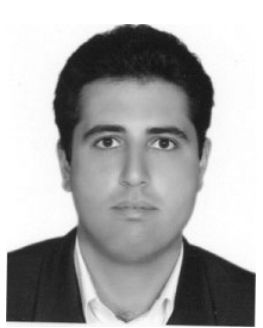

Abbas Taherpour (S07-M09) received the B.Sc. and $\mathrm{PhD}$ degrees in Electrical Engineering, Communication Systems, from Sharif University of Technology, Tehran, Iran in 2002 and 2009, respectively. He also received his M.Sc. degree from Tehran University, Tehran, Iran in Electrical Engineering, Communication Systems in 2004. He is currently an assistant professor with the Electrical Engineering Department of Imam Khomeini International University (IKIU), Qazvin, Iran. He has been a visiting scholar at Queen's University, ON, Canada form June 2007 until June 2008 and has participated in several wireless communications and signal processing projects at the regional and European level with industry and institutions. His research interests are cognitive radio, statistical signal processing and wireless communications. 